\title{
Structural and functional stabilization of protein entities: state-of-the-art is
}

\author{
Victor M. Balcão ${ }^{\mathrm{a}, \mathrm{b}, *}$, Marta M.D.C. Vila ${ }^{\mathrm{a}}$ \\ ${ }^{a}$ LaBNUS - Biomaterials and Nanotechnology Laboratory, i (bs) ${ }^{2}$ - intelligent biosensing and biomolecule stabilization research group, University of Sorocaba, Sorocaba, SP, Brazil \\ ${ }^{b}$ CEB - Centre of Biological Engineering, University of Minho, Braga, Portugal
}

\section{A R T I C L E I N F O}

Available online 12 October 2014

\section{Keywords:}

Structural and functional stabilization

Protein stability

Biopharmaceutical applications

Bacteriophages

Immobilization and post-immobilization

\begin{abstract}
A B S T R A C T
Within the context of biomedicine and pharmaceutical sciences, the issue of (therapeutic) protein stabilization assumes particular relevance. Stabilization of protein and protein-like molecules translates into preservation of both structure and functionality during storage and/or targeting, and such stabilization is mostly attained through establishment of a thermodynamic equilibrium with the (micro)environment. The basic thermodynamic principles that govern protein structural transitions and the interactions of the protein molecule with its (micro)environment are, therefore, tackled in a systematic fashion. Highlights are given to the major classes of (bio)therapeutic molecules, viz. enzymes, recombinant proteins, (macro)peptides, (monoclonal) antibodies and bacteriophages. Modification of the microenvironment of the biomolecule via multipoint covalent attachment onto a solid surface followed by hydrophilic polymer co-immobilization, or physical containment within nanocarriers, are some of the (latest) strategies discussed aiming at full structural and functional stabilization of said biomolecules.
\end{abstract}

(c) 2014 Elsevier B.V. All rights reserved.

\section{Contents}

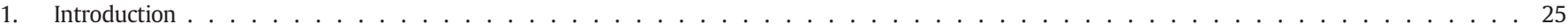

2. Protein entities with (bio)pharmaceutical application possibilities . . . . . . . . . . . . . . . . . . . . . . . . . . . . 28

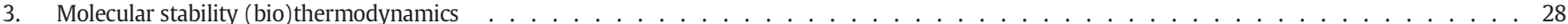

4. Thermodynamic stability . . . . . . . . . . . . . . . . . . . . . . . . . . . . . . . . 29

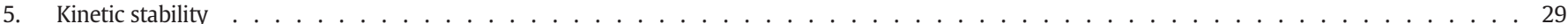

6. Structural and functional stabilization of monomeric and multimeric entities . . . . . . . . . . . . . . . . . . . . . . . . . . 30

6.1. Stabilization of protein entities via engineering at the level of the microenvironment . . . . . . . . . . . . . . . . . . . . . . 30

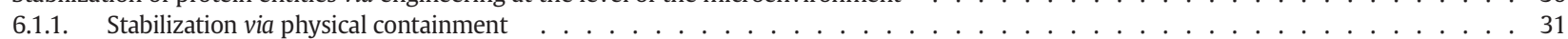

6.1.2. Stabilization via physical adsorption onto macroscopic supports . . . . . . . . . . . . . . . . . . . . . . . . . . . 32

6.1.3. Stabilization via chemical bonding onto highly activated supports . . . . . . . . . . . . . . . . . . . . . . . . 32

6.2. Stabilization of protein entities via engineering at the level of the macroenvironment . . . . . . . . . . . . . . . . . . . 35

6.2.1. Medium engineering . . . . . . . . . . . . . . . . . . . . . . . . . . 35

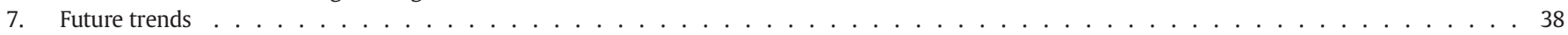

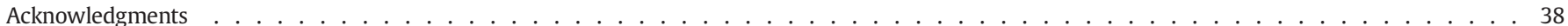

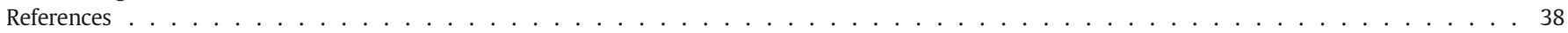

is This review is part of the Advanced Drug Delivery Reviews theme issue on "Protein stability in drug delivery applications".

* Corresponding author at: Universidade de Sorocaba (UNISO), Cidade Universitária Prof. Aldo Vannucchi, Rod. Raposo Tavares km 92.5, CEP 18023-000 Sorocaba, São Paulo, Brazil. Tel.: +55 152101 7181; fax: +55 1521017000 .

E-mail address: victor.balcao@prof.uniso.br (V.M. Balcão).

\section{Introduction}

In its essence, all life forms are polymeric, since their most important components (proteins, peptides, carbohydrates and nucleic acids) are all biopolymers. And nature uses polymers both as building bioblocks and as part of the highly complex cell machinery. While nowadays proteins and peptides are well known to be the not-so-secret secret of life, the real secrets and mystery of life are hidden in an extremely complex 
structure of natural biomacromolecules, viz DNA and RNA. Besides those naturally occurring (bio)polymers, humans now possess the technology to produce synthetic macromolecules via polymerization processes to form polymer-based artificial materials, usually aiming at replacing natural ones.

But both natural and synthetic polymers display a strong structureproperty relationship and, therefore, a detailed knowledge of the structure of macromolecules is of utmost importance in modern (bio)polymer chemistry and biotechnology. It is the fact that such biopolymers are ubiquitous in the metabolic machinery of all living beings, that makes them so much attractive for biotechnological and industrial applications. In fact, the lifetime of proteins inside the cells must be limited, since constitutive proteolysis is the main source of amino acids for de novo protein synthesis $[1,2]$. But we are interested in proteins in nonnative conditions for biotechnological applications.

Protein stabilization has a tremendous importance due to the increasing number of protein applications in almost all areas [3], but especially in the biopharmaceutical and biomedicine areas [4-7]. However, the only moderate stability of proteins, and specially enzymes, is the major drawback hindering the generalized application of these bioactive molecules at the industrial scale $[8,9]$. The causes of poor biocatalyst stability are closely associated with the process conditions prevailing, and may include extreme temperatures or $\mathrm{pH}$ values [10], or even the presence of organic solvents, that are outside the operating stability window of the biomolecule, but that are often necessary to solubilize poorly-water-soluble substrates in high concentration values [11]. In particular, within the context of biomedicine and pharmaceutical sciences, the issue of (therapeutic, recombinant) protein stabilization assumes particular relevance [4,9,12-15]. Protein instability is one of the major drawbacks that hinders the (more appealing) oral administration of protein pharmaceuticals [6,9].

When preparing and applying (biopharmaceutical) protein preparations for use in biomedicine, one usually faces three aspects of protein instability, viz. operational stability (dictated by the ability of the protein to survive processing operations such as sterilization of enzyme drugs $[6,7]$ ), thermodynamic stability (dictated by the ability of the folded protein conformation to resist denaturation during contact with different media (e.g. plasma, cytoplasm) and interfaces (e.g. cell membrane lipid bilayer) following oral, transdermal or intravenous administration), and in vivo stability (following penetration into the target cell) [2,3,16-18].

Stabilization of protein and protein-like molecules translates into preservation of both structure and functionality during storage and/or specific targeting, and such stabilization is mostly attained through establishment of a thermodynamic equilibrium between said molecules and their (micro)environment [19]. Therefore, to satisfy the increasing demand of biomolecules with biopharmaceutical applications, a basic understanding of the interactions between such biomolecules and their (micro)environment is in order. There is a delicate balance between stability and flexibility needed for enzyme function [20-25], added to the increasing awareness of the importance of the protein surface for stability $[1,3]$, since it is through this interface that a protein entity senses the "external world". It is generally accepted that functionally important amino acid residues are mainly solventaccessible residues on the protein surface, while structurally important residues are likely part of the protein core $[2,21,26]$. According to Jaenicke [27] and Vieille and Zeikus [28], the core packing in native (folded) protein molecules is so well arranged that virtually all solvent molecules are essentially excluded, making the protein core more like a crystalline solid than a non-polar fluid.

Damborsky [29] investigated the effect of the changes in structure of a protein on its function and stability. By using quantitative structurefunction relationship (QSFR) or quantitative structure-stability relationship (QSSR) analyses, Damborsky [29] aimed at investigating and mathematically describing the effect of the changes in structure (viz. amino acid modifications by site-directed mutagenesis) of macromolecules such as proteins on their activity, specificity or stability. Foit and colleagues [18], Becktel and Schellman [30], Somero [31] and Lee and Vasmatzis [32] extensively discussed the intrinsic stability of a protein molecule, i.e. the stability that can be obtained by mutating its amino acid residues, and concluded that by mutating some amino acid residues of the hydrophobic-core packing of a protein molecule, it is possible to (thermally) stabilize a protein molecule to a remarkable degree. Protein thermal stability usually increases if amino acid substitution and/or modification results in increased internal and decreased external (or surface) hydrophobicity [31,33-38]. Similarly, Campos and colleagues [39] used one of the most promisingly general methods of protein stabilization, the optimization of surface charge-charge interactions, a method which relies in identifying by theoretical methodologies, mutations of a protein that are expected to lower the electrostatic energy of the native state. In this way, charge (reversal) mutations involving the replacement by lysine of negatively charged residues not involved in either saltbridges or hydrogen bonds, and located at the protein surface, were introduced in the apoflavodoxin from Anabaena PCC7119 [39], and the resulting increase in stability of the native, folded state, duly verified relative to its unfolded counterpart.

More than $95 \%$ of all charged moieties are located on the surface of the protein [20], consisting mostly of hydrophilic moieties (while most of the hydrophobic ones are buried deep inside the core [30,40, $41]$ ), and since the static or dynamic conditions of the physicochemical microenvironment of the protein are sensed intrinsically through those moieties, the protein surface thus constitutes a very challenging and attractive target for protein engineering aiming at enhancing its stability $[37,42]$. In the same line of thought, Palmer and colleagues [43] mutated a protein-G (a cell-surface protein from Streptococcus) aiming at improving stability towards caustic alkali, demonstrating that strategies for stabilization of proteins at extreme alkaline $\mathrm{pH}$ should consider thermodynamic stabilization that will retain the tertiary structure of the protein and modification of surface electrostatics, as well as mutation of alkali-susceptible moieties (e.g. in their study, all three labile asparagine (Asn) residues within the IgG binding domain of protein-G were replaced/mutated). In a similar fashion, Villegas and colleagues [4] demonstrated that by using a helix/coil algorithm to design (secondary structure component) helix-stabilizing mutations on the (solely) solvent-exposed moiety patches of protein helices, it was possible to rationally increase the stability of proteins.

Bioactive entities such as insulin, (therapeutic) enzymes, fibrinogen, (recombinant) monoclonal antibodies, interferons, and bacteriophages, among others, are mainly produced via synthetic methods using biofactories such as cloned transgenic animals (cows, goats and ewe), cloned transplastomic plants, (recombinant) microbial fermentation and mammalian cell culture. Of these, (recombinant) monoclonal antibodies constitute the second largest biopharmaceutical product category currently in clinical trials. Highly complex production processes and, concomitantly, high production costs associated with such complex protein molecules, all require that they are fully stabilized and preserved at high efficiency levels during long periods of time. The structural stability of protein entities is extensively controlled by the interactions between the protein molecules and the surrounding solvent molecules [44]. Protein stabilization is based upon dampening the molecular motions and, therefore, eliminating conformational transitions while the molecule is still in the native (three-dimensional folded) state. A further, and especially complex problem, lies in the stabilization of multimeric proteins, with dissociation of the subunits producing enzyme inactivation with concomitant product contamination $[8,38,45]$. The stability of multimeric proteins is very dependent on the concentration, due to their multimeric nature, which translates into a low thermal stability under diluted conditions due to a subunit dissociation mechanism $[45,46]$.

Due to the high glutathione concentration inside the cell, there is no disulfide bond in intracellular proteins, hence many of the cross-links stabilizing the three-dimensional structure of proteins are clusters of 
non-covalent bonds [2]. The (folded) three-dimensional structure of a protein molecule depends mostly on two types of interactions: intramolecular interactions between amino acid moieties (hydrogen bonds, van der Waals interactions, hydrophobic interactions, disulfide bridges) and intermolecular interactions with solute and/or solvent molecules present in its environment (ionic bonds, hydrogen bonds, polar interactions) [6]. Makhatadze and Privalov [47], Liao et al. [48] and later Miyawaki [49], pointed out in their studies, the importance of intramolecular hydrogen bonding in the stabilization of proteins.

When looking at the (non-specific, indirect) effects of solutes on the molecular motions of proteins, one can envisage modification of the solvent promoted by the former at four levels: (i) molecular motions, (ii) chemical properties, (iii) physical properties, and (iv) thermodynamic properties. Water is the universal and natural solvent for proteins, dictating the molecular motions, the structure and function of these molecules [22]. Globular proteins are only marginally stable, and such metastability makes proteins difficult to handle experimentally [12,13, 18,50-53]. According to Bizzarri and Cannistraro [54], a threshold level of hydration $\left(0.4 \mathrm{~g}_{\text {water }} / \mathrm{g}_{\text {protein }}\right)$ is required to fully activate the functionality of globular proteins, an amount less than would be necessary for a complete coverage of the surface of the protein. Later on, we will address the issue of the essential hydration shell of all proteins, a role played beautifully by such universal solvent $[3,12,13]$. Proteins are strongly hydrated in aqueous solutions, and the hydration state affects their stability, function and three-dimensional conformation $[49,55]$. Since water is the environment in which protein molecules do exist and operate, the structure and dynamics of the water hydration shell are directly linked to both protein flexibility and stability $[22,24,56]$. Protein stability is directly correlated with the ability of water molecules in the hydration layer to fluctuate among different equilibrium structures and, additionally, internal water molecules contribute also to protein stability by providing the necessary flexibility for biological activity, by acting as lubricant and by rendering to the protein a certain level of plasticity $[22,24,57]$. Additionally, water can take part in the reaction indirectly by providing solvation to polar residues of the enzyme (or other protein) and other intervening molecules in order to facilitate protein conformational changes during the biocatalytic process [24].

A protein molecule can only move if the nearest molecular neighbors also move, a collective phenomenon resembling a continuous search for escape out of a cage rather than a discontinuous jump across an energetic barrier [57]. The (liquid) cage becomes a trap when the density reaches a critical value, a moment when the liquid is arrested on a macroscopic scale. Water, therefore, acts as a plasticizer to protein motions, expanding the accessible protein conformational space by decreasing friction [57], via changing allegiances of hydrogen bonds between donors and acceptors [58]. Structural plasticity is thus dominated by polar interactions [57]. Stabilizing a biomolecule involves dampening its molecular motions, and this can be achieved by reducing the chemical activity of the water present in its microenvironment at the expense of either (i) changing the thermodynamic phase of water, (ii) exposing the water to specific solutes (such as the disaccharide trehalose), or (iii) by completely removing such water (viz. by freeze drying). Dehydration thus leads to a virtually infinite viscosity, or glassy state [57], implying that embedding a protein molecule in a rigid solvent or removing the solvent entirely reduces small-scale liquid-like motions.

Proteins are characterized by a small thermodynamic stability $[16-18,30,44]$. The thermodynamic stability of a protein entity conformation is the result of several non-covalent interactions which may occur intramolecularly or with the solvent [44]. Thermodynamic stability of a protein is, in effect, the work required to disrupt the tertiary structure of the protein [51,59], where the transition from the native (folded) state to the denatured (unfolded) conformation is a highly cooperative process involving disruption of intramolecular hydrogen bonds, hydrophobic interactions and other types of non-covalent interactions [30,44,50,51]. In freeze-drying, as the water content decreases, hydroxyl groups from excipient (osmolyte) molecules might be expected to gradually approach and hydrogen bond with protein entities $[48,60]$, thus accounting for the preservation of the native structure of proteins. Stabilization mechanisms for preservation of viable cells (microorganisms, animal, plant, insect) also involve modification of the water within their microenvironment, via e.g. freeze drying with replacement of water molecules close to the membrane lipid headgroups with trehalose [60]. Ganjalikhany and colleagues [61] studied the effects of trehalose and magnesium sulfate on the structural stability and function of luciferase from firefly (Photinus pyralis), and noticed that the stability of this enzyme increased in the presence of the additives. They concluded that magnesium sulfate and trehalose can be used, respectively, for short- and long-term stabilization of the enzyme.

Kohda and colleagues [62] proposed an intriguing method for the (thermal) stabilization of immobilized enzymes, viz. the coimmobilization of hyperthermophilic chaperonin (molecular chaperones that play a central role in protein folding in vivo $[1,63]$ ) from Thermococcus strain KS-1. Hyperthermophilic chaperonins are expected to stabilize proteins because they are thermostable and suppress the thermal inactivation of enzymes [62-64].

Irrespective of the method being utilized, stabilization procedures aim at preserving the function of a protein by stabilizing both its structure and functionality during storage. Protein structures are composed of ordered regions, alpha-helices and beta-sheets, which are connected by disordered turns [57], and involve four different domains: primary, secondary, tertiary and quaternary.

The primary structure encompasses a specific linear sequence of amino acids that form the protein, and remains totally unaltered during preservation processes since the peptide (covalent) bonds between the amino acids are quite strong and are not broken by changes in hydration status or temperature. The secondary structure is the three-dimensional construct assumed by certain parts of the primary structure. These local constructs are mainly determined by hydrogen bonds that are established within the primary domain [65]. The two most common constructs encompassing the secondary domain are the $\alpha$-helix and the $\beta$-pleated sheet. The $\alpha$-helix is a right-handed coiled or spiral conformation, in which every backbone $\mathrm{N}-\mathrm{H}$ group donates a hydrogen bond to the backbone $\mathrm{C}=0$ group of the amino acid four residues earlier ( $i+4 \ni i$ hydrogen bonding). This secondary construct is also sometimes called a classic Pauling-Corey-Branson $\alpha$-helix [66]. Among types of local structure in proteins, the $\alpha$-helix is the most regular and the most predictable from sequence, as well as the most prevalent. The $\beta$-pleated sheet is the second form of regular secondary construct in proteins, only somewhat less common than the alpha helix. $\beta$ Pleated sheets consist of $\beta$-strands connected laterally by at least two or three backbone hydrogen bonds, forming a generally twisted, pleated sheet. A $\beta$-strand is a stretch of polypeptide chain typically 3 to 10 amino acid long with backbone in an almost fully extended conformation. The higher-level association of $\beta$ sheets has been implicated in formation of the protein aggregates and fibrils observed in many human diseases, notably the amyloidoses such as Alzheimer's disease. Generally, protein aggregation involves mostly $\beta$-sheets while $\alpha$-helix constructs seem to be less likely to form aggregates [60]. The denaturation and aggregation of proteins, observed during either preservation processes or storage, are often associated with changes in the populations of $\alpha$-helixes, $\beta$-sheets and random coil structures within the protein $[50,60,67]$.

From the industrial pharmaceutical biotechnology point of view, protein aggregation is undoubtedly the most common and troubling manifestation of protein instability encountered in almost all phases of protein drug development ( $v i z$. fermentation, isolation and recovery as inclusion bodies, unfolding-refolding, purification, formulation, storage), which hinders the rapid commercialization of potential proteinbased drug candidates [6,12,13,60].

The tertiary domain of a protein encompasses its three-dimensional folded-shape, with the hydrophobic side-groups of the amino acid moieties hidden within the core [41] and the hydrophilic groups being 
exposed to the surrounding microenvironment. In monomeric proteins, it is the tertiary domain of the protein that determines its function. The primary and secondary domains of a (monomeric) protein both contribute to its tertiary domain. Any changes occurring in the physicochemical and thermodynamic properties of the protein's microenvironment will affect the secondary and tertiary domains of the protein, therefore causing a change in its three-dimensional conformation, followed by unfolding (denaturation) with concomitant loss of function $[30,67,68]$. The secondary construct of a protein entity is generally more resistant to environmental aggressions compared to the associated tertiary domain [67]. The contribution of water activity was proved to be always positive for stabilization of proteins $[49,69]$, since lubricating protein motions appears to be the main role of water [57].

The quaternary domain is the combination of two or more monomeric chains (or subunits), to form a complete (multimeric) unit. The interactions between the monomeric chains are not different from those in the tertiary domain, but are distinguished only by being interchain rather than intrachain. Some (multimeric) proteins are composed of identical subunits (monomeric chains) (as in the case of the dimeric HIV Protease), while other proteins are composed of non-identical subunits (as in the case of insulin), which is made up of two chains, the $\alpha$ chain and the $\beta$-chain, linked by two disulfide bridges.

The three-dimensional spatial architecture of proteins is mainly determined by two classes of non-covalent interactions, viz. electrostatic and hydrophobic. Electrostatic interactions between polar and ionized groups include ion pairing, hydrogen bonds, weakly polar interactions and van der Waals forces. Hydrophobic interactions imply van der Waals forces and hydration effects of non-polar moieties [16,22,27]. The physical nature of the latter was recently interpreted as being entropic and enthalpic due to significant contributions from van der Waals forces $[22,51,70]$.

Whether we talk about native or recombinant proteins, all have in common the existence of three protein domains in the case of monomeric proteins, and the existence of a fourth protein domain in the case of multimeric proteins. As we shall see later, it is the third and the fourth domains the ones directly involved in the stabilization of monomeric and multimeric proteins, respectively. Very recently, Kong and $\mathrm{Hu}$ [71] reviewed a new area of research, that of bioactive paper fabrication through immobilization and stabilization of biomolecules for applications such as paper-based bioassays, antimicrobial paper with incorporated bacteriophages for food packaging, and bioactive paper with incorporated bacteriophage particles for pathogen detection. In the same line, Balcão and colleagues $[72,73,183]$ succeeded in fully stabilizing (both structurally and functionally) phage particles within hydrogel matrices, for bacterial pathogen detection.

\section{Protein entities with (bio)pharmaceutical application possibilities}

A protein entity can be conceptualized as being a molecule that is produced by a living organism, including large polymeric molecules such as proteins, macropeptides, polysaccharides, lipids, and nucleic acids as well as small molecules such as primary metabolites, secondary metabolites, and natural products. A more general name for this class of molecules is "biogenic substance", meaning a substance produced by life processes. With recent advances in biotechnology, protein and peptide drugs have become the center of current formulation research [14, 15,74-76]. Therapeutically significant peptides include, for instance, calcitonin, cyclosporine A, insulin, protein antigens (e.g. hepatitis B and malaria antigens). There are many examples of bioactive protein entities with biopharmaceutical application possibilities, viz. (therapeutic) enzymes, recombinant proteins and peptides, (monoclonal) antibodies and bacteriophages [6,15].

Proteins are therefore apparently inert biopolymers possessing cavities and crevices and, when we think of a very special class of proteins, lipolytic enzymes, we can also observe adsorption and hyper-reactivity characteristics. But, either native or recombinant (i.e. artificially produced using biotechnological platforms with cloned genes), all protein entities exhibit a three-dimensional structure that holds hydrophobic residues in the inner core and which is held by means of faint links and interactions such as hydrogen bonds between peptide bonds, saline bridges, hydrophobic interactions, van der Waals interactions and disulfide bridges. $[50,65,67,77,78]$. Enhanced stability in proteins results from changes in the protein core, which are mostly attributed to increased burial of hydrophobic groups from aqueous solvent $[28,78]$, providing greater solvation energy favoring folding.

Biotherapeutics, whether using recombinant proteins, (engineered) antibodies, enzymes, (macro)peptides or latest developments such as RNA interference (RNAi), allows addressing specific targets not so much susceptible to small molecule approaches. Bioactive peptides have been defined as specific protein fragments that have a positive impact on body functions or conditions and may ultimately influence health. Upon oral administration, bioactive peptides may affect the major body systems, namely the cardiovascular, digestive, immune and nervous systems. The beneficial health effects may be classified as antimicrobial, antioxidative, antithrombotic, antihypertensive, antimicrobial or immunomodulatory [14].

Bacteriophages, or phages ... what are they? These are a special kind of viruses that uniquely and exclusively infect bacterial cells. They are obligate parasites, requiring a host microorganism to multiply and spread and, although carrying all the genetic information to direct their multiplication within permissive host cells, do not possess the metabolic machinery needed to produce energy, neither ribosomes for protein synthesis. They are, therefore, completely inert and highly advantageous both as antimicrobial entities and in bacterial pathogen biosensing devices [72,73,183].

However, both proteins and peptides, and phages, are prone to denaturation via conformational changes that can be either reversible or irreversible. Not very high temperatures, $\mathrm{pH}$ values not close to the extremes [10], chaotropic agents (urea and guanidinium hydrochloride) and hydrophobic interfaces, all may promote partial unfolding of the native (folded) three-dimensional architecture and, if we remove them, re-naturation of the protein entity is in principle promoted [3]. However, if the unfolding process is too extense, promoted by an excessive breaking of the interactions that maintain the tertiary structure, one can have permanent inactivation. From this event on, chemical modifications and the action of proteases, as well as intermolecular aggregation of completely unfolded molecules, all promote permanent denaturation $[3,6,60]$. The solution lies in protecting both proteins and peptides, and phages: by encapsulating such biomolecules within nanocarriers, eventually endowed with characteristics of invisibility towards the digestive and immune systems, or binding them to macroscopic supports thus rendering them insoluble. Combined, these strategies promote their structural and functional stabilization $[72,73$, 118,183].

\section{Molecular stability (bio)thermodynamics}

At the molecular level, a protein molecule may oscillate between many (slightly) different three-dimensional conformations [19,22]. Each oscillation between two conformations is ruled by the second law of thermodynamics, viz. $\Delta \mathrm{G}=\Delta \mathrm{H}-\mathrm{T} \Delta \mathrm{S}$, where $\Delta \mathrm{G}$ is the difference between the Gibbs free energy of the two three-dimensional conformations, $\Delta \mathrm{H}$ is the transition enthalpy, $\Delta \mathrm{S}$ is the transition entropy, and $\mathrm{T}$ is the absolute temperature $[1,50,79]$. The conformational Gibbs energy depends on the contribution of conformational enthalpy and mainly on the enthalpy of intramolecular hydrogen bonds and on the van der Waals interactions between all the protein atoms [79]. In these transitions between configurations, with the protein molecule seeking the lowest energy state possible, if the value of $\Delta \mathrm{G}$ is negative the protein molecule would denature.

Application of the mathematical expression of the second law of thermodynamics assumes, naturally, that the native (folded) and the 
denatured (unfolded) states are unique states corresponding to unique three-dimensional configurations of the protein moiety. However, in their works, Sanchez-Ruiz [7], Ragoonanan and Aksan [19], Scharnagl and colleagues [22], Shenoy and Jayaram [23], Becktel and Schellman [30], Doster and Settles [57], and Shah and colleagues [67] have shown that a protein can assume a very high number of slightly different configurations (or microstates) both in the native and in the denatured states, among which the protein molecule continuously oscillates through bond vibrations, throwing thermodynamics of protein transitions into a highly complex energy landscape. Equilibrium of a protein moiety in any of these states is dictated both by entropic and enthalpic factors, and therefore stability theories predict that proteins can denature not only by increasing the temperature but also by decreasing it (called cold denaturation) [19,22,80,81]. The total entropy of the protein molecule together with the associated water shell decreases as the protein folds, while the enthalpic contribution is mostly due to hydrogen bond formation [22].

Protein stability is, as mentioned above, quantitatively described by the standard free Gibbs energy change involved in unfolding the unique, three-dimensional structure, to a randomly-coiled polypeptide chain [1, 80,82,83]. According to Miyawaki and Tatsuno [69] and Khechinashvili [79], the Gibbs energy of the hydration process of protein unfolding is a large and negative value. While crosslinks of inert polymers increase stability entropically by decreasing the entropy gain on unfolding or dissociation, disulfide bonds in proteins can increase stability also by increasing the transition enthalpy [82].

In their studies, Zhou et al. [84] showed that molecular mobility directly determines the storage stability of amorphous pharmaceutical compounds. Although proteins are relatively large molecules, they are not infinite systems. There is, therefore, a direct relationship between molecular motions of the protein moiety and the molecular motions of its immediate neighborhood, which implies that stabilization can eventually be achieved by dampening any motions of the microneighborhood (thereby increasing protein's rotational, translational and vibrational viscosity). Since the protein moiety is constantly exploring a multitude of configurational landscapes [1] while, at the same time, interacting with the solvent molecules and other surrounding solutes, it can adopt configurations that favor intermolecular interactions, virtually resulting in the formation of hydrophobic interactions among protein moieties (aggregation) [22,60,85]. Enzymes are thus devices, which select by construction a small fraction of events out of a large number of fast structural fluctuations [6,57], selecting the most stable three-dimensional architecture which is the one with the lowest energy.

Denaturation of a protein moiety involves unfolding of its threedimensional architecture, in a way that biological or biochemical activity is lost [3]. Chemical agents such as chaotropes (urea or guanidinium hydrochloride) can also lead to denaturation [86,87]. In high-viscosity, low-molecular mobility systems, molecular mobility and denaturation kinetics have been shown to be coupled [88]. All these processes may affect the stability of protein entities during both preservation processes and storage [67]. According to Khechinashvili and coworkers [81], the thermal stability of proteins does not correlate with the energy of intramolecular interactions, and so the mechanism of thermal stabilization of a compact protein with an ordered structure is largely of an entropic (rather than energetic) nature. During the denaturation process of a protein molecule induced by temperature, the protein molecule changes from a rather well organized structure into a random coil structure in which the hydrophobic amino acid moieties (once buried inside the three-dimensional structure of the protein) come into intimate contact with water. As a consequence of this intimate contact, water molecules form locally ordered structures around the hydrophobic amino acid moieties (forming the so-called icebergs, in a thermodynamically unfavorable process) which are characterized by both a low entropy and a low enthalpy, due to the well-ordered hydrogen bonds [22].

\section{Thermodynamic stability}

(Protein) thermodynamic stability relates to protein stability to reversible changes of structure in non-native in vitro conditions [7,16,30, 67]. The biological function of a protein is guaranteed if equilibrium is established between the native and the (partially) unfolded states of the protein [59]. One can judge the stability of any protein structure by studying its disruption and, since a protein molecule is a macroscopic system, the disruption of its structure can be thought of as a change of the macroscopic state of the system [80]. The (thermodynamic) stability of a protein molecule is usually expressed as Gibbs energy, since $\Delta \mathrm{N} G$ is the work required for disruption ( $\mathrm{D}$, disrupted) of the native $(\mathrm{N}$, globular) protein structure $[6,43,59,80,89-91]$. Therefore, this difference between the Gibbs energies of the native and completely random states serves as a measure of protein stability [79], and the larger this value the more stable the protein is [6]. While the Gibbs energy of hydration of nonpolar groups, though a relatively small value, shifts the imbalance of forces towards the formation of the native structure [79]. According to Privalov [80], the native state of a protein is most stable at the temperature where the entropy difference of the native and denatured states is equal to zero, and it is stabilized only by the enthalpy difference of these states.

Understanding how the biopolymers of life adapt to their microenvironments is central to devise thermodynamic stability. The rationale is that protein stability can be partially understood by examining the $\alpha$ helices in the three-dimensional architecture and their constituent amino acid residues. One of the principal factors that controls $\alpha$-helix stability is the presence of intra-helical, non-covalent bonding interactions, such as hydrogen bonds. Such interactions exist in thermodynamic equilibrium and, therefore, their strength will be strongly influenced by physical factors of the environment [22]. Imprisonment of a protein entity leads to thermodynamic stability, which can be correlated with a change in the thermodynamic conditions of the microenvironment surrounding each bioparticle, since the movements of (aqueous) solvent molecules in their microneighborhood become seriously reduced by the effect of being contained within the matrix's core. The result is that the protein entity's rotational, translational and vibrational viscosity becomes enhanced, leading to a more rigid three-dimensional architecture with concomitant decrease of entropy and producing stabilization.

\section{Kinetic stability}

Long-term or operational stability characterizes a protein's ability to resist irreversible changes of structure in in vitro non-native conditions $[2,3,7,16,18,48,92,93]$. Kinetic stability is a measure of how quickly a protein unfolds, hence measuring the resistance to irreversible inactivation $[3,17,92,93]$, often being expressed as the protein's half-life $\left(t_{1 / 2}\right)$. It can be considered a "long-term stability". In the case of irreversible unfolding, it is the kinetic stability or the rate of unfolding that assumes particular importance. When irreversible alterations of non-native states occur very fast, the rate of irreversible denaturation is given by the rate of unfolding $[92,93]$.

A kinetically stable protein entity will unfold slower than a kinetically unstable one. In a kinetically stable protein entity, a higher free energy barrier $(\mathrm{Ea})$ is necessary for the unfolding process, and the factors that affect stability are the relative free energies of the folded $\left(G_{F}\right)$ and transition state $\left(\mathrm{G}_{\mathrm{TS}}\right)$ (critically activated) conformations [7]. A protein entity can denature irreversibly if its unfolded form undergoes a permanent quick change such as aggregation and proteolytic degradation [3, $16,60,67]$. For the former change, it is the patches of contiguous hydrophobic groups in the folding-unfolding intermediates that initiate the aggregation process $[1,60]$, because mutual attraction of hydrophobic moieties and/or patches (which results in aggregation) minimizes the area of unfavorable protein-solvent interface. 
Proteins aggregate to minimize thermodynamically unfavorable interactions between solvent and exposed hydrophobic residues $[6,44$, 50]. Hence, it is not the difference between the free energies of folded and unfolded states that is important; such difference only affects equilibrium. Truly important is, as stated, the difference between free energies of folded and transition states (Ea), since it is the magnitude of this difference that determines the unfolding rate (and therefore, of inactivation). Besides the biotechnological implications for biopharmaceutical industry [6,23], there is a general interest in understanding protein kinetic stability since some emerging molecular approaches to the inhibition of amyloidogenesis focus on the increase of kinetic stability of protein native states [42,92].

\section{Structural and functional stabilization of monomeric and multimeric entities}

The term "protein stabilization" refers to preservation of the unique chemical and three-dimensional structure of a polypeptide chain under extremes of physical conditions. Stabilization of multimeric enzymes and proteins in general represents a especially complex problem, if we realize that among the most interesting enzymes with potential biopharmaceutical applications many of them are of multimeric nature $[8,45]$. As mentioned above, in monomeric proteins the first step in their inactivation involves generally alterations in the tertiary structure (i.e. in the three-dimensional architecture) $[33,94]$. However, for multimeric problems, inactivation begins generally either with dissociation of the enzyme subunits or with alterations in their correct threedimensional assembly [8,95-97]. But the multimeric nature of these protein entities does not translate at all into a low stability. On the contrary, the quaternary architecture resulting from the multiple subunits assembly provides a decrease in the surface area accessible, which in turn has been associated with extreme thermophilicity $[8,70,82$, 98-100]. Quaternary interactions and closer packings are typical characteristics of proteins from thermophiles [6,101]. In multimeric proteins, subunit-subunit multi-interactions also contribute to improve protein rigidity due to a lower mobility of the moieties involved in these interactions.

Notwithstanding the fact that multimeric proteins are intrinsically more stable than monomeric ones due to their rigidity when compared to their monomeric counterparts [3], under certain experimental conditions such subunit-subunit multi-interactions may be weakened leading to dissociation of subunits and concomitant rapid inactivation of the multimeric protein [8]. Hence, prevention of subunit dissociation is the first goal when stabilization of such highly interesting protein entities is sought $[8,45,95-97,102-107]$.

When we speak of enzymes, and particularly multimeric enzymes, their structural and functional stabilization assumes special relevance through immobilization and post-immobilization techniques [11,45, $65,96,97,108-110]$, because as well as being necessary to ensure rigidification of the molecule it is also necessary to stabilize it against the action of denaturing organic solvents [24,111]. Protein stability inversely correlates with its flexibility, with the flexible patches in proteins typically being the labile areas $[6,28,31,112]$. A folded protein in an aqueous environment (solution) has hydrophobic regions sequestered from, and hydrophilic areas in contact with, the aqueous environment. But, when the polarity of an aqueous solvent decreases via addition of a nonaqueous solvent, protein hydrophobic cores tend to dissipate in contact with the latter which leads to disruption of the protein hydration shell, with concomitant destabilization and unfolding of the macromolecule $[6,51,69]$.

In terms of structural stabilization, its rigidification occurs via intense multi-subunit covalent immobilization, but maintenance of its functional stability occurs only after cross-linking all of its subunits $[45,96,113]$, which promotes additionally its stability in the presence of organic solvents by maintaining the three-dimensional architecture of the enzyme perfectly hydrated due to creation of a hyper- hydrophilic nanoenvironment. When structural rigidification (with concomitant three-dimensional architecture stabilization) is sought, multipoint covalent immobilization into a highly activated support is the preferred immobilization strategy. It is possible to structurally stabilize proteins by sacrificing activity to a certain degree [36,109,114]. An ideal support should be characterized by a subtle balance between the affinity towards the protein entity (providing the driving force for protein binding) and the absence of critical structural distortions (affecting the protein stability and activity) [115].

Stabilization of proteins and similar molecules translates into preservation of both structure and functionality during storage and/or targeting, with such stabilization being attained mainly through establishment of a thermodynamic equilibrium with the (micro)environment. Protein stabilization is based on elimination of molecular motion(s) and, therefore, on the elimination of conformational transitions while the biomolecule is still in its native (folded) threedimensional architecture [52]. A bioactive molecule (protein, peptide, phage particle) encapsulated in the core of a nanovesicle becomes an independent phase within a food/pharmaceutical formulation, with the advantage of being protected and having its useful active life extended. Encapsulated biomolecules can therefore be considered as being localized in a given defined region of space, limited by an imaginary or physical barrier that allows physical separation between the biomolecule and the surrounding food/pharmaceutical formulation, and between the biomolecule and the immune/digestive system.

Immobilization of a biomolecule can then be achieved via engineering the microenvironment or engineering the macroenvironment of the biomolecule [116]. Structural stabilization is related to threedimensional rigidification of the biomolecule, while functional stabilization involves maintenance of the hydration shell of said biomolecules and, in the case of multimeric proteins, preservation of the quaternary assembly $[96,97,113,117]$. All current stabilization mechanisms of protein entities in terms of chemical modification are depicted in Fig. 1. Hence, to achieve full stabilization of a protein entity, irrespective of its number of subunits, this will always involve two levels of stabilization: structural stabilization and functional stabilization. These will be tackled detailedly below.

\subsection{Stabilization of protein entities via engineering at the level of the microenvironment}

Engineering at the level of the biomolecule's microenvironment includes immobilization by physical containment in a barrier (viz. microencapsulation within lipid vesicles or reverse micelles, entrapment within polymeric matrices, nanoencapsulation [72,116,118]), or establishment of covalent bonds to a macroscopic support. However, the immobilization of an enzyme (or other protein) does not guarantee per se the stabilization of the enzyme structure [119]. Thermal stability generally results from the molecular rigidification introduced by attachment of the protein molecule onto a solid support with concomitant creation of a protected microenvironment [3,111].

Crosslinking proteins in solution to form oligomeric microstructures without any macroscopic carrier is a strategy used to increase protein activity per unit area, providing enhanced solvent- and thermal stability [65]. Cross-linked enzyme aggregates (CLEAs, obtained by precipitation of the protein followed by cross-linking) allow engineering of the microenvironment $[3,24,37,65,117]$ through the co-aggregation of enzymes and polymers (for example, to reduce solvent interactions or to reduce oxygen dissolution). However, CLEAs are mechanically fragile and present extense diffusional limitations in mass transfer, hence they should then be encapsulated within LentiKats ${ }^{\mathrm{TM}}$, so as to gain mechanical resistance [120-122]. Several different enzymes have been stabilized using this approach, as reviewed by Talbert and Goddard [65], who extensively reviewed all the changes occurring upon contact of enzymes with material surfaces. 


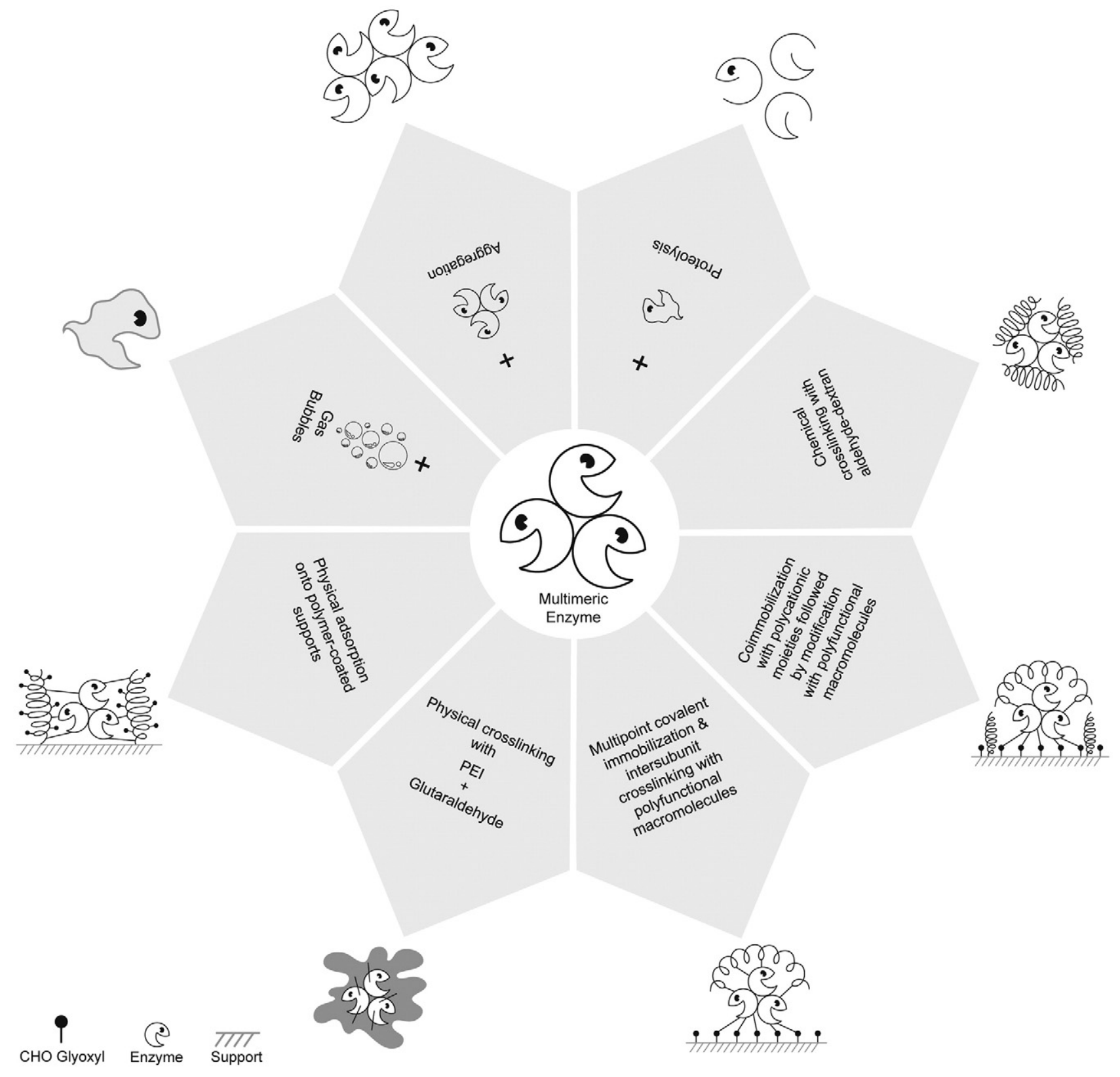

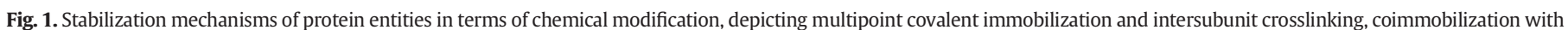
polycationic moieties, chemical crosslinking with aldehyde-dextran, and physical adsorption onto polymer-coated supports.

Protein stabilization can also be achieved via covalent modification $[16,87]$, thus improving some properties of the protein surface (e.g. charge, polarity, hydrophobicity). Such properties are especially important for in vivo stability, by determining, for example, (i) protein distribution between tissues, and (ii) the ability to penetrate into different cellular compartments $[18,23]$. Several approaches can be used to enhance protein stability in vitro by covalently modifying surface functional groups [16,37], viz. (i) protein surface modification with bifunctional reagents to crosslink surface functional groups (with e.g. glutaraldehyde, diimidates, or disulfonyl chlorides), (ii) chemical modification of the protein with nonpolar reagents to enhance hydrophobic interactions, and (iii) protein modification with hydrophilic reagents to promote formation of additional hydrogen or ionic bonds, since the more hydrophilic groups are introduced in the molecule the greater the stabilizing effect $[3,16]$. Other approaches for the chemical modification of proteins include grafting to either polysaccharides (e.g. dextran activated by S-triazine and/or sodium metaperiodate, or mono-6-amino-6- deoxy-beta-cyclodextrin) or polymers (e.g. polyvinylpyrrolidone $(\mathrm{PVP}))[3,25]$. Fuentes and colleagues [123] reported on the successful immobilization of IgG anti-horseradish peroxidase onto magnetic nanoparticles previously coated with aldehyde-aspartic-dextran, aiming at structural stabilization of the antibodies with proper orientation on the support.

\subsubsection{Stabilization via physical containment}

The thermodynamic stabilization of protein molecules is particularly important when it comes to nanocontainment as a way to convey this type of biomolecules. Within this context, nanoencapsulation procedures within lipid nanovesicles have started to gain momentum and assume now special relevance $[72,118]$. By providing a hydrophilic core for the imprisonment of the protein entities, lipid nanovesicles make their rigidification possible since they greatly limit the molecular movements around the trapped bioentities. They limit, in particular, the movements of solvent molecules co-entrapped in their core. Lipid 
nanovesicles are, therefore, ideal for the immobilization by physical containment of protein entities, promoting their thermodynamic stabilization. In the process of their production, when forming multiple emulsions, the poloxamer used promotes formation of a threedimensional network favorable to the maintenance of imprisonment of the protein entities.

The advantages of protein nanoconfinement are several: (i) localization of the biomolecules in a given, defined, portion of space, (ii) enhanced thermal and chemical resistance, (iii) ease of application by the improved resistance transmitted to the biomolecules, as well as (iv) potential for stabilization at room temperature [24,118]. The stability of protein entities following their nanoconfinement can be described both in thermodynamic and kinetic terms. Thermodynamically, protein stability comes from two large but opposing forces, viz. enthalpic and entropic. Both of these forces are temperaturedependent. The enthalpic forces (the intramolecular interactions) are stabilizing, and the entropic forces (the dissipative forces, loss of conformational entropy of the native state) are destabilizing [34].

Thermodynamic (or conformational) stability can be correlated with a change in the thermodynamic conditions of the microenvironment surrounding each bioparticle, since the movements of (aqueous) solvent molecules in their micro-neighborhood become seriously reduced by the effect of being contained within the matrix's core. Since there is a direct relationship between the molecular movements of the biomolecule and the molecular motions of its immediate neighborhood, when we encapsulate a biomolecule we are eliminating the motions of the solvent molecules in its immediate neighborhood (thus increasing biomolecule's rotational, translational and vibrational viscosity) [118].

The Reynolds number $(\mathrm{Re})$ is a dimensionless value that gives us a measure of the ratio between inertial and viscous forces, thus quantifying the relative importance of these two types of forces under certain flow conditions $[180,181]$. We live in a world where Reynolds numbers are very high due to the presence of high inertial forces and very low viscous forces. However, at the micro-scale, viscous forces are by far the dominant forces, and therefore Reynolds numbers at the microscale are very low. Hence, at the micro-scale, fluid mixing occurs by simple molecular diffusion due to the absence of both molecular turbulence and inertial forces. In the interior of vesicles, Re is null, while in their exterior Re is higher than zero. Since the encapsulated protein entities are to be used in some kind of formulation, with flow properties, Re values can also account for the stabilization produced by the encapsulation procedure due to the absence of turbulent flow in their inner core [180,181].

Confinement of a protein entity in a nanoporous matrix has a similar effect as that of osmolytes with respect to changing the water activity and thus modifying biochemical reaction rates. Within such nanoenvironments, the motions of water molecules are so much restricted that they do not crystallize even at temperatures close to the absolute zero but instead, transition into a highly viscous state, stabilizing the protein entities by hyper-increasing its rotational, translational and vibrational viscosity $[8,22,64,83,124]$. Confining multimeric proteins within liposomes have been proposed as a general strategy to stabilize such protein entities [125]. Additionally, reverse micelles (closed, almost spherical aggregates of surfactant molecules (e.g. AOT) with the outer shell formed by the hydrophobic tails and polar heads forming the inner core) can be seen as a two-phase system, with enzymes encapsulated therein exhibiting very high activities when compared with their native (soluble) counterparts [3].

\subsubsection{Stabilization via physical adsorption onto macroscopic supports}

Enzyme immobilization by physical adsorption traditionally refers to binding of the enzyme via weak attractive forces to an inert carrier which has not been chemically derivatized. Since the carrier is directly involved in binding to the enzyme, its chemical nature, particle size (in the case of granules) or thickness (in the case of membranes), and pore size distribution (especially in the range where pores are large enough to be accessible to enzyme) play important roles [116,126]. In general, physical adsorption of an enzyme is achieved by simply contacting the buffered enzyme solution with the carrier, which may require minimal pretreatment such as wetting, washing, and presoaking in the buffered solution (especially when the carrier possesses hydrophobic character) and usually requires minimal post-treatment such as washing out the excess solution containing nonadsorbed enzyme. Adsorption of proteins (including enzymes) at solid/liquid interfaces is of great technical significance. Particularly in the case of lipases, hyper-activation of these enzymes has been observed upon contact with hydrophobic (solid) interfaces that generally mimic the presence of oily substrates [127-129], due to the so-called "interfacial activation" phenomenon $[115,130]$.

The use of physical supports previously coated with very large and flexible ionic polymers may allow the immobilization of multimeric enzymes via multi-subunit interactions, providing that the enzyme molecule can penetrate into such flexible coating and interact with the polymers [131,132]. Additionally, several researchers [133] have proven that the selective physical adsorption of a multimeric protein, viz. glutamate dehydrogenase from Thermus thermophilus, onto a very lowly activated (heterofunctional amine and agarose) support (via the lowest amount of amine groups) followed by covalent immobilization (via the dense layer of reactive epoxy groups) could promote immobilization of the enzyme by the maximum amount of subunits and attain rigidification of its subunits involved in the immobilization. These authors found out that glutamate dehydrogenase immobilized on a support possessing at the same time a low density of amine groups and a high density of epoxy groups, after incubation at pH 8.5 during 3 days did not release a single enzyme subunit. Vaidya and Singhal [134] used epichlorohydrin to convert the free hydroxyl groups in insoluble yeast $\beta$-glucan into activated epoxy groups capable of forming irreversible covalent bonds with various groups on the surface of Candida rugosa lipase, thus considerably improving its structural and functional stability with concomitant operational stability in non-aqueous medium. In the same line, Boscolo and colleagues [115] reported on the structural and functional stabilization of a lipase from Pseudomonas fluorescens following its physical adsorption onto cyclodextrin nanosponges, while Serno and colleagues [135] comprehensively reviewed the use of cyclodextrins for the stabilization of proteins both in the liquid and dried states.

\subsubsection{Stabilization via chemical bonding onto highly activated supports}

Ideal supports for the immobilization and concomitant rigidification of multimeric protein entities involve large concentrations of available glyoxyl groups in such supports. This makes it possible to establish multipoint covalent attachment between (non-ionized amine groups from) protein entities and the (highly reactive glyoxyl groups on the) support $[111,114,136]$. If stabilization is performed in solid phase (using immobilized enzymes, see Fig. 1), there are intrinsic added advantages in the sense that there is a greater control of the chemical modification because undesirable protein-protein interactions are minimized [117]. Immobilization of proteins improves both thermodynamic and operational stability [16], since these are mainly determined by the ability to withstand protein unfolding. The most important feature of (globular) protein unfolding is the exposure (to the solvent) of its hydrophobic core [41], with a volume change negative and very large in magnitude [52]. Therefore, an increase in both thermodynamic stability and operational stability should correlate with an increase in rigidity promoted by immobilization $[53,65]$, which in turn correlates with the number of bonds established between protein and support. However, the thermodynamic behavior of proteins in the process of "order-disorder" cannot be explained if the hydration of hydrophobic amino acid moieties from the protein molecule is not taken into account $[44,79]$.

In the chemistry of protein immobilization there are two fundamental steps: multipoint covalent attachment followed by reduction of the immobilized protein derivative (see Fig. 2). This allows to structurally 
stabilize the protein through its permanent rigidification, without major distortions in its structure [117]. A higher stabilization may be explained because of an optimal geometrical congruence among protein and support surfaces [111], together with a high surface density of reactive groups on the support. Two types of active groups especially important for this purpose are the glutaraldehyde groups ( to establish unipoint covalent bonds at $\mathrm{pH} 7.0$ with the terminal amine group of the protein), and the glyoxyl groups (to establish multipoint covalent bonds at $\mathrm{pH} 10.0$ with amine groups of the highest surface density of lysin moieties in the protein molecule, thus promoting increased structural stabilization) $[8,45,110,114,129,137,138]$. This explains the high enzyme stabilization usually achieved by using this immobilization technique $[45,129,138,139]$. López-Gallego and colleagues [137] also reported on the immobilization of several enzymes (viz. glutaryl-7aminocephalosporanic acid acylase, D-amino acid oxidase and glucose oxidase) via adsorption onto a cationic support (amino MANAEagarose) followed by crosslinking with glutaraldehyde, which allowed a remarkable stabilization of such covalently immobilized enzymes.

The use of enzymes (or other proteins) stabilized via multipoint or multi-subunit immobilization also limits the extent of their inactivation that may arise from chemical modification processes [109,114,117,182]. Cowan and Fernández-Lafuente [117] recently described the immobilization/stabilization of a series of thermophilic enzymes, viz. thermophilic amylases and xylanases, thermophilic sugar isomerases, thermophilic redox enzymes, thermophilic glycosidases, and thermophilic lipases and esterases.

Of all stabilization strategies, immobilization and post-immobilization techniques possess additional advantages due to allowing the enzyme (or other protein entity) to be reused for several cycles, thus increasing enzyme productivity [11,110]. Similarly, Serra and colleagues [11] reported on the immobilization of homodimeric thymidine phosphorylase from Escherichia coli via ionic adsorption onto amine-functionalized Sepabeads ${ }^{\mathrm{TM}}$ coated with polyethyleneimine, followed by cross-linking with aldehyde-dextran (MW of $20 \mathrm{kDa}$ ).

6.1.3.1. Multipoint covalent immobilization. Protein unfolding can be inhibited mechanically by covalently attaching it to a macroscopic support through multiple covalent bonds, thus promoting its rigidification. Agarose highly activated with glyoxyl groups has been described as a suitable support for the multipoint covalent immobilization of both monomeric and multimeric enzymes, allowing their full structural stabilization via attainment of a high degree of rigidity $[45,96,97,110,114$, $119,129,138,139]$. Multipoint immobilization of multimeric proteins may prevent subunit dissociation by inter-subunit crosslinking (see Fig. 1) while simultaneously reducing conformational inactivation by intra-subunit crosslinking $[45,46,113,129,140]$. The highly reactive aldehydes from glyoxyl react with amino groups from the surface of the proteins to form reversible imino bonds.
Proteins become immobilized on glyoxyl supports through a simultaneous multipoint bonding $[8,96,97,114,129,139]$, and this occurs via the richest Lys region of the protein surface at alkaline $\mathrm{pH}$. Despite this, proteins displaying several terminal amine groups (as is the case of multimeric proteins) with a $\mathrm{pK}$ in the range of $\mathrm{pH} 7-8$ will become immobilized on glyoxyl agarose even at $\mathrm{pH} 7$ via multiple subunits, as long as those amine groups are located in such a position that their simultaneous interaction with a flat surface becomes possible. Having in mind the extraordinary features of glyoxyl supports so stabilize proteins $[45,96,97,114,129,138,139]$, immobilization of multimeric proteins on these supports at pH 7 can be coupled to immobilization and stabilization via multisubunit and multipoint covalent attachment. Hence, following the first attachment at $\mathrm{pH} 7$ via the terminal amine groups of multiple subunits located in the same plane, the (already) immobilized molecules can be subsequently incubated at $\mathrm{pH} 10$ for longer periods of time to allow an intense multipoint covalent immobilization onto the support. After this time period, the protein derivative (set of support plus protein immobilized on the support) may be reduced with sodium borohydride (see Fig. 2) so as to re-convert the unreacted aldehyde groups back to inert hydroxyl groups while, at the same time, fully reducing the reversible Schiff's bases to irreversible covalent bonds [46] (see Fig. 2), yielding irreversible secondary amine bonds between the protein or the support, and the polyfunctional aldehyde-dextran polymer (in the case of inter-subunit cross-linking) [45,129], thus reducing the aldehyde groups present in the aldehyde-dextran to a highly hydrophilic and inert polyhydroxyl. However, some decrease in activity may be observed during the reduction process, which might be ascribed to the change of the reversible bonds formed during immobilization (Schiff's bases between the enzyme amino groups (mostly surface Lys moieties) and the support aldehydes) to irreversible ones (by chemical reduction of imino bonds to stable covalent carbon-nitrogen bonds), thus fixing the position of the protein residues relative to the support surface $[11,110]$.

In the copolymerization method of enzyme immobilization, the enzyme is first modified with a compound having a double bond, and then is copolymerized with a monomer and bifunctional crosslinking reagent producing a three-dimensional polymeric network [16]. Immobilization of therapeutical enzymes by copolymerization is extensively used for biomedical uses [3]. However, some of the disadvantages of enzyme immobilization are related to diffusional problems and activity losses due to the somewhat harsh conditions prevailing during the immobilization process [3]. Additionally, López-Gallego and colleagues [119] reported on the multipoint covalent attachment of glutaryl-7-aminocephalosporanic acid acylase (an enzyme widely used in the pharmaceutical industry for the synthesis of 7-aminocephalosporanic acid from cephalosporin-C) onto amino-epoxy Sepabeads ${ }^{\mathrm{TM}}$, allowing to achieve a high degree of stabilization of the enzyme since in addition to Lysine moieties (which react mostly with glyoxyl groups), also Tyrosines have reacted with
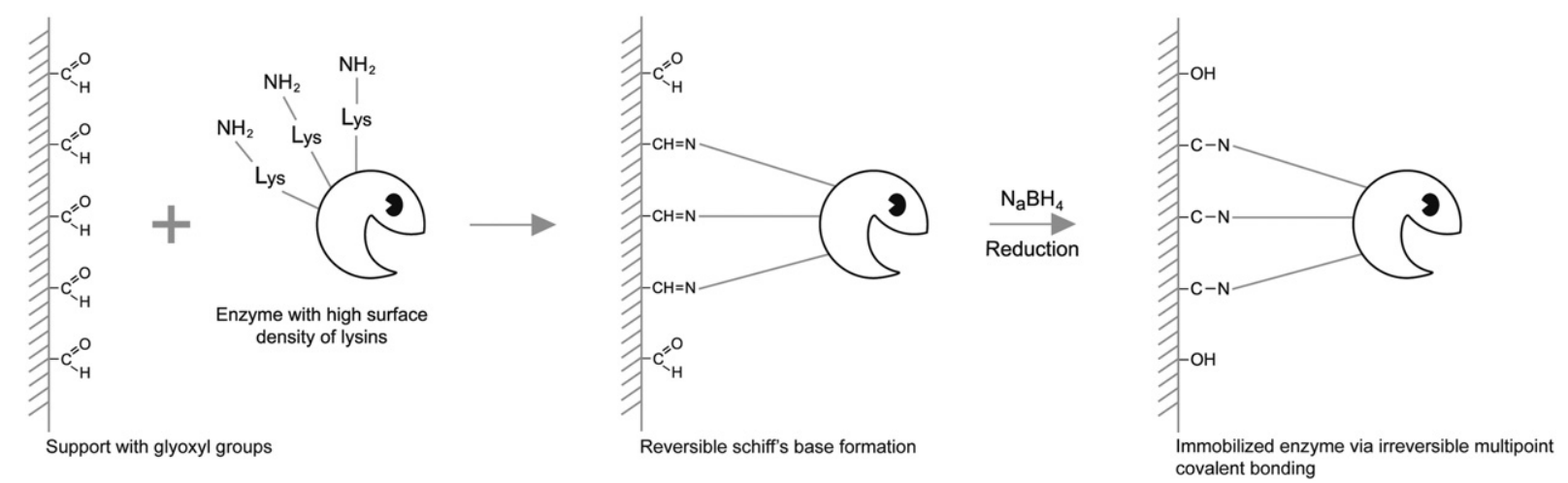

Fig. 2. Chemistry of protein immobilization via multipoint covalent attachment onto highly activated supports, followed by reduction of non-used reactive groups. 
the support. In the same way, López-Gallego and colleagues [46] reported on the structural and functional stabilization of several different multimeric alcohol oxidases (whose quaternary structure ranges from four to eight identical subunits three-dimensionally arranged in a quasi-cubic spatial distribution) via covalent immobilization onto glyoxyl-agarose followed by post-immobilization via intersubunit cross-linking with aldehyde-dextran to prevent subunit dissociation. Similarly, Bernal and colleagues [114] reported on the multipoint covalent immobilization of beta-galactosidase from Bacillus circulans onto hierarchical macro-mesoporous silica previously modified with glyoxyl groups, aiming at thermal stabilization of said enzyme for the production of prebiotic galacto-oligosaccharides (GOS). Those researchers [114] found out in their studies that the degree of thermal stabilization attained was affected by the concentration of glyoxyl groups on the surface of the porous silica particles, in a directly proportional fashion (up to a certain degree), probably because the enzyme rigidification attained is insufficient at low concentrations of glyoxyl groups for producing thermal stabilization.

However, the excessive rigidification of the protein molecule also leads to destabilization of the tertiary structure of the enzyme. According to Grazu and colleagues [111], a lowering in the number of the attachments protein-support may hamper an optimal rigidification of the protein tertiary structure, avoiding a high final stability of the immobilized protein.

6.1.3.2. Co-immobilization with polycationic moieties. Through coimmobilization with polyfunctional macromolecules (see Fig. 1), full stabilization of any protein entity (whether being monomeric or multimeric) will be possible from the point of view of its structure (rigidification) or of its functionality (hydration plus maintenance of the quaternary assembly in the case of multimeric proteins), by modifying the microenvironment of such immobilized molecules, which allows a tremendous increase of their stability in organic media [113,141]. Additionally, co-immobilization of flexible polycationic polymers (such as polyethyleneimine) (see Fig. 1) directly in the support allows a strong and non-distortive immobilization of the biomolecule, with the added advantage of facilitating the removal of the biomolecule and substitution for another (eventually different) one. Fernández-Lafuente and colleagues [142] attached Penicillin G acylase to glyoxyl agarose via multipoint covalent attachment followed by modification with ethylenediamine to increase free amine groups on the surface and intramolecular crosslinking of the amine groups with bifunctional glutaraldehyde, thus attaining increased stability to urea. Furthermore, Grazu and colleagues [111] succeeded in fully stabilizing Penicillin G acylase from $E$. coli by site-directed immobilization onto commercial monofunctional epoxy-acrylic supports, viz. Eupergit $C \circledR$, a type of support able to form very stable covalent linkages with different reactive groups on protein surfaces (primary amine, thiol, aromatic hydroxyl, carboxy, imidazoline).

Polymer conjugation may also be used to change the surface of an enzyme [65]. Modifications typically use polymers or small molecules to attach to the surface of an enzyme with the most common being carbohydrates and PEG. Such molecules are believed to provide additional points for hydrogen bonding with the enzyme surface, decreasing dehydration or providing thermodynamic barriers to unfolding [65]. Minimization of any interactions between the protein entity and (hydrophobic) solvent or gas bubbles (see Fig. 1) can proceed via grafting a polyhydrophilic (macro)polymer over the surface of the protein entity, thus creating a stable nanohydrophilic environment around the protein [141]. López-Gallego and colleagues [46] also employed another stabilizing immobilization protocol for multimeric alcohol oxidase enzymes, viz. the ionic adsorption on agarose coated with a polymeric bed of $600 \mathrm{kDa}$ polyethyleneimine, with the adsorption of such large proteins onto the large polymeric bed allowing full stabilization of the quaternary structure of said multimeric enzymes.
6.1.3.3. Further inter-subunit crosslinking with polyfunctional hydrophilic macromolecules. Fernández-Lafuente [8] and Cowan and FernándezLafuente [117] provided an extense and specific review on stabilization of multimeric enzymes, in particular with regard to strategies aiming at preventing subunit dissociation. Chemical modification is used to generate covalent crosslinking bonds between different groups on the enzyme surface (see Fig. 1); whether such crosslinking occurs between different structural elements (subunits) of a protein, it will typically amplify the structural rigidity and hence increase protein stability against (physical or chemical) agents that induce conformational changes (such as heat and chaotropic agents) [117].

Both physical and chemical crosslinking with poly-ionic macromolecules have been reported as a means to fully stabilize multimeric proteins. In physical crosslinking, the multimeric protein is coated with a poly-ionic macromolecule, such as polyethyleneimine (PEI) [141,143], followed by further treatment with glutaraldehyde (which crosslinks the enzyme and the poly-ionic macromolecule). Although preventing both subunit dissociation and other phenomena such as oxidation and aggregation, this strategy does not however promote rigidification of the multimeric structure. In intersubunit chemical crosslinking (see Fig. 1), aldehyde-dextran has proved to be suitable for fully stabilizing multimeric enzymes from the functional point of view $[8,25,45,96,97$, $113,117,141,144,145]$. Such polymeric multifunctional reagents have effectively prevented dissociation of multimeric proteins or multiprotein complexes [45,96,97,113,133,138,143].

When, due to geometrical constraints, it is not possible to immobilize all enzyme subunits onto a planar support, as is the case for example of tetrameric L-asparaginase from $E$. coli or hexameric bovine liver glutamate dehydrogenase [96,97], hexameric alpha-galactosidase from Thermus sp. T2 [113], glutamate dehydrogenase from $T$. thermophilus [133], or multimeric (eight subunits) microbial alcohol oxidases from Candida boidinii, Hansenula sp., or Pichia pastoris [46], coupling multisubunit and multipoint covalent attachment (thus stabilizing the maximum amount of enzyme subunits) to subsequent intersubunit crosslinking between covalently immobilized and non-immobilized subunits using polyfunctional hydrophilic macromolecules such as aldehyde-dextran, allows to prevent dissociation of the whole set of subunits composing the multimeric protein, thus allowing to stabilize the quaternary structure of these enzymes [45]. Such rigidification of the multimeric structure implies a full degree of structural stabilization of the protein. It should, however, be noted that once subunit dissociation is no longer a problem, inactivation will proceed via distortion of the protein three-dimensional structure by altering either the assembly of monomeric subunits or the structure of the individual subunits. Pessela and colleagues [113] have reported on the successful structural and functional stabilization of a multimeric (hexameric) enzyme (viz. alpha-galactosidase from Thermus sp. strain T2) via covalent immobilization onto cyanogen-bromide agarose (thus promoting its rigidification) followed by chemical modification with aldehyde-dextran aiming at inter-subunit crosslinking (thus promoting maintenance of the quaternary assembly of the enzyme). Also, Kotzia and Labrou [146] reported on the successful stabilization of L-asparaginase from Erwinia chrysanthemi 3937 onto epoxy-activated Sepharose ${ }^{\mathrm{TM}} \mathrm{CL}-6 \mathrm{~B}$ using 1,4-butanediol diglycidyl ether as a coupling agent. Similarly, Bolivar and colleagues [110] successfully immobilized a multimeric alcohol dehydrogenase from Baker's yeast on glyoxyl-agarose in the presence of acetyl cysteine, followed by inter-subunit crosslinking with (polycationic) polyethyleneimine (crosslinking the enzyme subunits via ionic interactions thus stabilizing the quaternary structure of the multimeric enzyme).

Polyethyleneimine is advantageous because due to its polymeric nature, it is likely to interact with areas on the protein surface located in different enzyme subunits, and is therefore desirable in the case of multimeric enzymes [11]. A new and effective methodology to covalently immobilize multimeric and sensitive enzymes at close to neutral $\mathrm{pH}$ values has been described [147], involving the use of thiolated 
compounds (such as N-acetyl-L-cysteine) during the immobilization process, providing the opportunity to immobilize highly sensitive enzymes via highly reactive glyoxyl groups but under very mild processing conditions. However, immobilization via glyoxyl chemistry at neutral $\mathrm{pH}$ does not stabilize the quaternary structure as it does the tertiary structure $[45,110]$. Thus, polyethyleneimine is highly suitable for use in the post-immobilization stabilization of the quaternary structure of multimeric enzymes that are very sensitive to covalent rigidifications $[11,110]$, by allowing rigidification of the tertiary structure (see Fig. 1).

\subsection{Stabilization of protein entities via engineering at the level of the macroenvironment}

Engineering on the level of the macroenvironment of the protein entity may proceed via modification of the reaction medium (e.g., precipitation in an organic solvent, by changing the rheology of the food/ pharmaceutical formulation matrix, or by addition of additives) aiming at modifying the surrounding environment $[12,13,116]$. The threedimensional architecture and molecular motions of a protein entity are determined to a large extent by the properties of its macroenvironment. Intrinsic characteristics of the macroenvironment where the protein entity is embedded, such as $\mathrm{pH}$, pressure, temperature, presence of salts and surfactants [8,19,22,24,51,87,148,149], all interfere with the structural and functional stability of the protein entity. Hence, stabilization methods via engineering at the level of the macroenvironment aim at modifying the thermodynamic state in the immediate vicinity of protein, either by cooling, freezing, vitrification, or removing the medium (as in the case of desiccation and freezedrying) [48].

From the thermodynamic point of view, the contribution of salts to a biocatalytic process is almost entropic and related to the degrees of conformational freedom along the transition states, with no enthalpic role $[24,150]$. According to the generally accepted Lumry-Eyring mechanistic model [151], enzyme inactivation is considered a two-step process: $\mathrm{N} \leftrightarrow{ }^{\mathrm{K}} \mathrm{U} \rightarrow{ }^{\mathrm{k}} \mathrm{I}$, where $\mathrm{N}$ represents the native form of the enzyme, $\mathrm{U}$ represents the reversibly unfolded form of the enzyme, I represents the irreversibly inactivated form of the enzyme, $\mathrm{K}$ represents an equilibrium (thermodynamic) constant of the reversible conformational change, and $\mathrm{k}$ represents the (kinetic) rate constant of the irreversible inactivation reaction $[3,6,7,17,44,92]$. Since it is easier to characterize the reversible conformational change of the protein, due to being highly sensitive to changes in $\mathrm{pH}$, ionic strength, chaotropes, among other additions and/or changes in the reaction medium, one can vary the value of $\mathrm{K}$ by changing the medium composition, which will directly affect the value of $\mathrm{k}$. In this way, the operational stability of the protein can be tailored [16].

\subsubsection{Medium engineering}

The structural stability of protein entities is extensively controlled by the interactions between the protein molecules and the surrounding solvent molecules [44]. Various substances were found to stabilize the native structure as a reflection of their effect on the water structure around the protein molecule [44]. Since it is almost impossible for the protein entity to retain its native configuration while its micro- and/or macroenvironment is modified during any process that involves cooling, freezing or desiccation, certain polyol moieties are added into the solution (viz. glycerol, trehalose, sucrose, maltose, dextran, DMSO, PVA, among others) [31,44,152-154]. All these chemicals share a common feature, in that they modify both the structure and motions of water molecules in the immediate vicinity of the protein entities $[8,19$, 24,83].

Irrespective of the exact mechanism of protection offered by such chemical moieties added to the solution, the immediate vicinity of the protein entity is modified, which reflects in the physical, thermodynamic and chemical properties of the surrounding solution. However, most cryoprotectant agents used (e.g. DMSO) are extremely cytotoxic (and even mutagenic) at high temperatures, and so their immediate removal should be pursued after thawing $[19,83]$.

6.2.1.1. Sugar and salt addition. Hydrophobic interactions, which can be regarded as the reluctance of non-polar moieties to be exposed to water, are considered to be the major driving force for both protein folding and aggregation $[6,28,44]$. Addition of polyols and sugars to aqueous solutions of proteins promotes strengthening of the hydrophobic interactions among non-polar amino acid residues, leading to protein rigidification and enhancing thermostability [3,5,6,12,13,34,48,89-91,154]. According to Miyawaki and Tatsuno [69], Haque et al. [90,91] and Kumar et al. [154], the effect of polyols on the activity of water seems to be the main factor that governs the stabilization effect produced by such polyhydroxy moieties.

There are two postulated explanations commonly employed to describe the mechanism of action of chemical moieties added to the reaction medium, through which both the molecular motions and structure of protein entities are affected: (i) the "water replacement" hypothesis, and (ii) the "preferential exclusion" hypothesis $[12,13,22,40,48,49$, 154-158]. The special ability of sugar moieties to bind protectively to the surface of biological molecular structures has been ascribed to their ability also to form hydrogen bonds [158]. Since the threedimensional architecture of virtually all protein entities in solution depends on stabilization of said architecture by a shell of water molecules hydrogen-bonded to their surfaces [3], the former hypothesis aims at explaining the protective effect of certain sugars against damage promoted by freezing and desiccation [19,48,159-163], predicting that the stabilization moieties added replace the water molecules that are removed from the hydration shells of the protein entities, thus stabilizing their native state; the later hypothesis states that the protective moieties are excluded from the surfaces of the protein entities and thus the available water molecules in solution can interact preferentially with the protein entity, thus stabilizing its native configuration $[5,19,49$, 164-166]. Due to the preferential exclusion of the protective sugar moieties from the (immediate domain) hydration shell of protein entities, sugar moieties shape a protective and stabilizing shield around those biomolecules $[5,154-157,167,168]$. The basis of this phenomenon is the difference in size between molecules of water and those of the sugar moieties. Essentially, a shell is formed around the protein at the radius of closest approach between the protein and the sugar moiety, a shell that is impenetrable to the sugar moieties but is penetrable to water, resulting in an excess of water in the vicinity of the protein. This is termed preferential hydration [12,13,155,167]. Preferential hydration of proteins is favored due to stronger interactions between sugar and water molecules compared to those between sugar and protein molecules [12,13,64,155].

Protein hydrophobicity increases with a decrease in $\mathrm{pH}$ due to the protonation of COO- groups, and so the increased exclusion of sugar moieties from the immediate vicinity of the unfolded protein molecule as compared with its native counterpart results in a larger stabilization effect at low pH values [168]. Xie and Timasheff [40] and Kaushik and Bhat [169] arrived at the same conclusion for ribonuclease-A in the presence of trehalose. Such preferential exclusion (i.e. preferential hydration of the protein) increases the chemical potential of the protein molecule [12,13,90,91,152,154,167] proportionally to the solvent exposed surface area and, according to the Le Chatelier's principle, sugar osmolytes favor the more compact state (viz, the native, folded state, F) over the structurally expanded state (viz. the unfolded, denatured state, U), which leads to an increase of the Gibbs free energy change associated with the denaturation process $(F \Leftrightarrow U)$ in the presence of osmolytes (one should remember at this point that $\left.\Delta \mathrm{G}_{D}=-\mathrm{RT} \ln ([\mathrm{U}] /[\mathrm{F}])\right)[7,18$, 30,52,67,69,89-91,156,168,170].

Such increase in the chemical potential of the protein is thermodynamically unfavorable $[90,91,171]$, meaning that protein interaction with the osmolyte is unfavorable relative to water, and so the protein 
is preferentially hydrated and the osmolyte is preferentially excluded from the protein surface $[6,167,170]$. Typically, this enhancement of stability increases with both increasing sugar concentration and increasing sugar molecular size $[12,13,55,154,157,168]$. According to Arakawa and colleagues [12,13], Wong and Tayyab [64], Timasheff and Arakawa [167] , and Hall and Minton [172], the generalized exclusion of (added) carbohydrate molecules from the protein surface (i.e., preferential hydration of the protein) might be explained by the molecular crowding concept [153], with steric exclusion, increase of the surface tension of water by the added moieties, repulsion by charged loci on the protein and solvophobicity.

The interactions of carbohydrate moieties with protein molecules progressively inhibit, with increasing extent of crowding, any conformational change of a protein that increases its effective volume, such as protein unfolding $[153,157,172]$. The sugar's concentration near the protein surface is lower than in the bulk, and so any process (such as unfolding) that increases the protein's solvent-exposed surface area is disfavored because exclusion of the sugar from a larger surface area entails a correspondingly larger free energy cost [157]. In particular, in the case of multimeric entities, these become thermodynamically stabilized against stress-induced dissociation [8]. Since protein unfolding increases the total surface area [49], in the presence of preferentially excluded moieties the free energy of unfolding is increased, and so preferentially excluded moieties can also stabilize quaternary structures because the combined surface area of the dissociated subunits is greater than that for the fully assembled protein entity [8].

The unfolding process of a protein in solution can be described by the Tanford's equation, $\mathrm{N}+\Delta \mathrm{i} \mathrm{W}+\Delta \mathrm{j} \mathrm{Y} \rightarrow \mathrm{U}$, where $\mathrm{N}$ is the native form of the protein, $\mathrm{W}$ is the water, $\mathrm{Y}$ is the co-solute moiety to bind protein upon unfolding, $\Delta \mathrm{i}$ is the change in hydration number per protein molecule, $\Delta \mathrm{j}$ is the change in the bound co-solute molecules per protein molecule, and $U$ is the unfolded form of the protein $[69,173]$. Osmolytes (such as sucrose or glycerol) stabilize proteins since they do not directly interact with (bind to) the protein molecules [3,16,22, $44,83,148,156]$, being excluded from their surface. This is a sine qua non condition for maintenance/stabilization of biological function. Such qualitative correlation between preferential osmolyte exclusion and protein stabilization is expected from the thermodynamic consequences arising from preferential interactions [6,40,83,90,91].

Changing the microenvironment of a protein via addition of sugars, polyols, salts, polymers, among others, leads to inhibition of protein aggregation and concomitant stabilization of these macromolecules via preferential interactions $[6,12,13,40,44,60,89-91]$. In this way, added polymers might inhibit protein aggregation via several mechanisms, viz. surface activity, preferential exclusion, steric hindrance of proteinprotein interactions and increasing viscosity thus limiting protein structural movements. Lavelle and Fresco [87] found, in the same way, that protein stabilization by high concentrations of salts follows the Hofmeister series. Also, certain surfactants (e.g. poloxamers) may also increase the viscosity of a protein solution, restraining the motions of the protein backbone to inhibit protein aggregation and induce thermal stabilization $[6,60,149,174]$. However, it should be stressed that these thermodynamic arguments hold true only when osmolyte interactions with the protein are affected by increased surface area upon unfolding. Since the unfolded state of protein molecules has a greater surface area than the native state [49], it should exclude more co-solute molecules leading to a more negative preferential interaction parameter for the unfolded state than for the native state and a greater increase in the values of $\Delta G[83,90,91]$ and in turn to stabilization of the protein molecules by the co-solutes.

Excipients are generally required to increase long-term stability of protein entities following processing and storage $[12,13,48]$. The thermodynamic interaction of osmolytes with protein molecules therefore stabilizes the equilibrium state of the (more folded) native or nativelike protein structure $[83,49,156]$. These molecules stabilize proteins not by interacting with them directly, but by altering the solvent properties of the surrounding water and therefore the protein-solvent interactions $[90,91,156,175]$. Trehalose is a non-reducing (polyhydroxy) disaccharide composed of two residues of D-glucopyranose (alpha-D-glucopyranosyl( $1 \rightarrow 1$ )-alpha-D-glucopyranoside), and is able to increase the stability of the folded conformation of proteins [40,49,55,61,69,152,158,169].

Sugars in general protect proteins against dehydration by hydrogen bonding to the dried protein by serving as water substitute $[12,13,48$, 169] (see Fig. 3), increase the thermal unfolding temperature and inhibit irreversible aggregation of protein molecules [44,154]. The interaction of sugar moieties with the protein reduces the contact area of hydrophobic fragments in the protein with water (see Fig. 3), leading to a decrease in free energy of the system and concomitant stabilization of the protein molecule [6]. Magnesium sulfate acts as a stabilizer, and its stabilization property is determined by a competition phenomenon between (i) salt exclusion effect and (ii) salt binding effect. The stabilizing salts seem to increase surface tension at water-protein interface and strengthen hydrophobic interactions by keeping hydrophobic moieties away from water molecules, inducing preferential hydration of proteins $[6,34,87,89,167]$.

When in presence of a stabilizing osmolyte, a protein entity prefers to interact with water molecules (preferential hydration), with the osmolyte being preferentially excluded from the protein domain (preferential exclusion), hence proportionally more water molecules and less osmolyte molecules are found at the protein surface than in the bulk solution $[5,6,90,91]$. By increasing the surface tension of solvent, salt exclusion of sulfate anion (preferential hydration) results, whereas the salt binding effect refers to the magnesium cation affinity for ionic residues and peptide bonds [61]. According to Wimmer and colleagues [5], the most widespread mechanism behind exclusion of polyols (such as sorbitol) from the protein surface lies in its enhancing effect on the surface tension of water. Arakawa and colleagues $[12,13]$ reviewed in detail the factors affecting short-term and long-term stabilities of proteins. The long-term stability of firefly luciferase [61] in the presence of additives showed that trehalose has a positive effect on storage stability (i.e. long-term stability) of this enzyme at refrigeration temperature, whereas magnesium sulfate has a positive effect on its short-term stability (i.e. thermal stability). Similarly, Miyawaki [49] and Miyawaki and Tatsuno [69] studied the contribution of the water activity of several sugar solutions (sucrose, trehalose, glucose, maltose, and ribose) for the (positive) stabilization of proteins (viz. ribonuclease A, lysozyme and $\alpha$-chymotrypsinogen).

In comparison to sucrose and trehalose, maltose, glucose and ribose are reducing sugars, interacting with proteins by forming a Shiff-base between the reducing terminal aldehyde of the sugar and the amino group of proteins at high temperature, explaining at least partially the incomplete precipitation of proteins in the presence of such (reducing) sugars $[49,55]$. Haque and colleagues $[90,91]$ studied the effect of several polyol osmolytes (D-sorbitol, D-glycerol, D-mannitol, D-adonitol, and D-xylitol) on the stabilization of RNAse-A and egg white lysozyme, having concluded that the secondary and tertiary structures of the native proteins remained unchanged upon addition of the aforementioned polyols. In contrast to magnesium sulfate, trehalose cannot dissociate into ion species, but it can increase the solvent viscosity which in turn enhances the preferential hydration of the enzyme $[90,91,158,169]$ and can affect the structural dynamics and decrease the molecular collisions of the protein molecules [61]. Such high viscosity causes a motional inhibition that hinders processes leading to the loss of structure and denaturation [158].

Glycerol, on the other hand, lowers the surface tension of water and has been hypothesized to preferentially hydrate protein molecules by enhancing the solvent ordering around the hydrophobic groups of the protein molecules $[12,13,154,176]$. Such ordering results in a decrease in the entropy of water present in the immediate hydration shell surrounding protein molecules [31], shifting the equilibrium towards the native (folded) state and producing stabilization of the protein molecule 

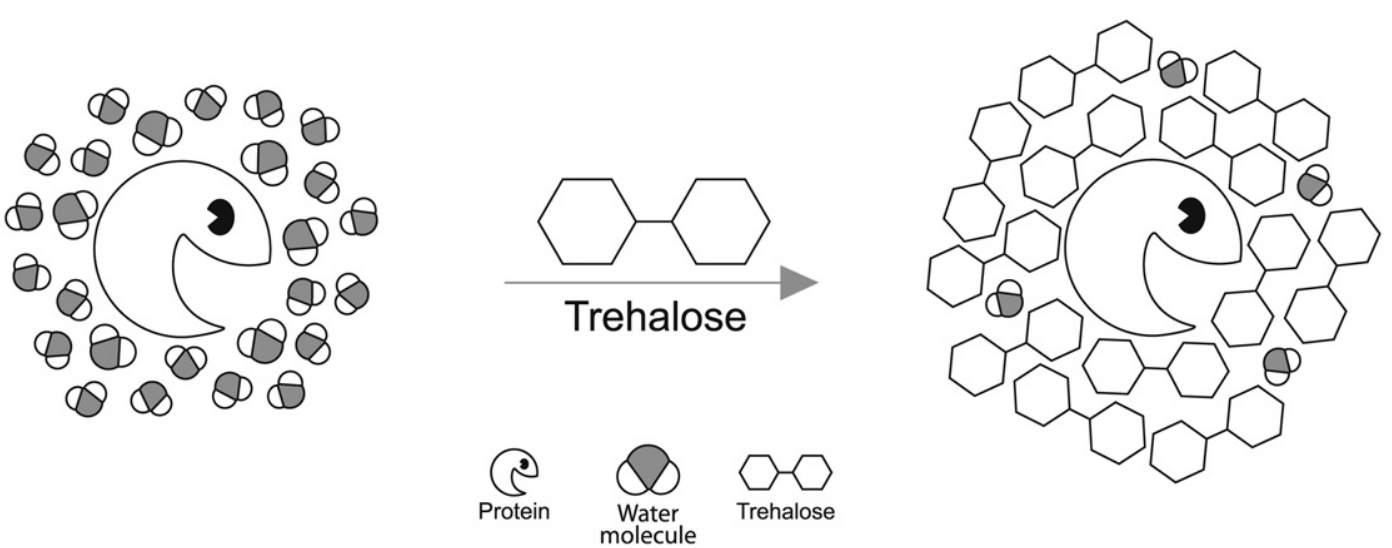

Fig. 3. Mechanism of protein stabilization by (disaccharide) trehalose.

$[12,13,64,154]$. Any increase in the hydrophobic surface area of protein entities upon unfolding would thus be rendered even more unfavorable in the presence of glycerol [154]. Liao and colleagues [48] reported on the stabilization of lysozyme following freeze-drying in the presence of trehalose, sucrose, or dextran. On the contrary, due to a high frequency of protein molecular collisions in the presence of magnesium sulfate, aggregation occurs at higher temperatures, due to the exposure of hydrophobic patches to solvent $[44,60]$. Miyawaki and Tatsuno [69] and Kaushik and Bhat [169] have studied the mechanism of trehalosemediated thermal stabilization of a set of globular proteins (viz. ribonuclease A, lysozyme, cytochrome $c$, alpha-chymotrypsinogen, and trypsin inhibitor), and concluded that trehalose in addition to imparting thermodynamic stability to proteins also helps in the retention of activity of enzymes during storage at high temperatures. According to Xie and Timasheff [40], Kumar et al. [154], Anjum et al. [156] and Kaushik and Bhat [169], trehalose stabilizes proteins by shifting the equilibrium constant (Native $\Leftrightarrow$ Denatured) in favor of the native state, and that it is the smaller preferential binding to the unfolded protein than to the native one which gives rise to the stabilization $[40,156]$. O'Connor and colleagues [157] found out that, at the same molar concentration, the disaccharide sucrose stabilizes proteins (viz. alpha-lactalbumin and ribonuclease-A) against thermal denaturation to a greater extent than does the monosaccharide fructose, suggesting that geometric differences between carbohydrates play a predominant role in determining their relative stabilizing ability, because the larger sugar (sucrose) makes a greater contribution to the unfavorable entropy of interface formation between protein and solvent. According to O'Connor and colleagues [157], the increase in protein stability observed in the presence of sugars is primarily due to the increase in the free energy of creating the protein-solvent interface. Poddar and colleagues [168] also studied the thermal denaturation of ribonuclease-A in the presence of glucose, fructose, galactose, sucrose, raffinose and stachyose, and concluded that an oligosaccharide has more stabilizing effect than the individual monosaccharide constituents, and also that on the same molar scale the order of stabilization is stachyose $>$ raffinose $>$ sucrose $>$ glucose $=$ fructose $=$ galactose .

Another postulate, the "water entrapment hypothesis", states that sugars concentrate residual water molecules close to the surface of the biomolecule, thus enabling the biomolecule to preserve its solvation/ hydration layer and native properties [158]. In their studies, Liao and colleagues [48] found out that the combination of either trehalose or dextran with sucrose as lyoprotectants resulted in an equivalent protective capacity to sucrose alone and was better than either trehalose or dextran alone, in the stabilization of lysozyme. Since water plays a crucial role in the establishment of hydrogen bonds between protein molecules and the medium, ions mimicking the water role in accepting or donating hydrogen bonds facilitate conformational changes and functioning of bioactive proteins such as enzymes [24]. Additionally, the effect of kosmotropic ions on the water surrounding hydrophobic residues of the protein has been reported to be associated with an increase in the order of water molecules while, at the same time, enhancing the strength of the hydrophobic interactions providing a more closed protein shell structure with concomitant increase in stability [177].

Eriksson and colleagues [178] studied the effect of both inulin and trehalose in the stabilization of bovine intestine alkaline phosphatase (BIAP) in tablets, and concluded that inulin was by far superior to trehalose as stabilizer of BIAP, with the poor stabilizing capabilities of trehalose after compaction being explained by both crystallization of trehalose induced by the compaction process and moisture in the material. According to Wang [6], when selecting sugars for protein stabilization reducing sugars should be avoided, since these sugars have the potential to react with the amino groups in proteins via the Maillard pathway. According to Arakawa and colleagues [12,13] and Eriksson and colleagues [178], stabilization of proteins via addition of a sugar followed by lyophilization or spray-drying is attained because the protein is incorporated in a matrix consisting of amorphous sugar in its glassy state, which imparts a strong reduction in the mobility of the protein molecule, and this has in fact been referred as the main factor for protein stabilization. Wong and Tayyab [64] reported on the stabilization of bovine serum albumin following addition of a simulated (devoid from protein and peptide interferents) honey sugar cocktail. According to these researchers, honey may be a potential natural stabilizer for proteins due to the presence of a high sugar content in it.

6.2.1.2. Addition of glycerol, DMSO, PEG, and other synthetic polymers. Stabilizing agents are inert substances that modify the physicochemical properties of aqueous solutions used to preserve biomolecules. DMSO and glycerol are preferentially excluded from contact with the protein surface, which means that the protein molecule is preferentially hydrated, thus accounting for their cryoprotective effect $[3,12,13,155,158]$. The stabilizing effect of PEG (an amphipathic polymer) addition increases with both increasing PEG concentration and PEG chain length $[3,179]$, which is related with the promotion of increased solvent ordering in the medium.

The stabilizing effects of polymer addition into the reaction medium may be explained by the fact that the polymer promotes exclusion of the protein molecule from part of the solvent and, in this way, prevents detrimental effects of the environment upon the protein molecule [3]. Shulgin and Ruckenstein [179] reported also that upon addition of PEG into the medium, protein molecules become preferentially hydrated, which could be explained by a steric (hindrance) exclusion mechanism due to the large difference in sizes between water and PEG molecules, with PEG being excluded from the vicinity of the protein molecule. It is thus established the ability of a cosolvent to stabilize a protein molecule, because the preferential hydration of a protein in an aqueous solution containing an organic compound is related to the 
ability of the latter to stabilize the structure of the protein $[12,13,55,155$, 179]. These authors [179] also concluded that the excess number of water molecules around a protein molecule increases monotonically with increasing PEG molecular weight, which is in agreement with the aforementioned steric (hindrance) exclusion mechanism.

\section{Future trends}

Combinatorial approaches to protein stabilization such as multipoint covalent immobilization followed by co-immobilization with flexible polycationic polymers and subsequent intra- and inter-subunit crosslinking with polyfunctional hydrophilic macromolecules and additional coating with aldehyde-dextran or (polycationic) polyethyleneimine have been used as strategies for the structural and functional stabilization of both monomeric and multimeric enzymes $[45,96,97,110,113]$. Together with nanocontainment strategies $[72,73$, 118], all these techniques for the structural and functional stabilization of protein entities can be thought of as protein engineering tools. But more and more, techniques aiming at the full stabilization of protein entities based on nanoencapsulation procedures started to gain momentum.

Due to being entrapped in very confined environments, the molecular motions of water molecules are altered due to their dipolar nature, causing them to slow down. Confinement in nanoporous matrices or nanocapsules has, therefore, the same effect as the osmolytes with respect to changing the water activity. Due to increased viscosity (both translational, vibrational and rotational) and entropic confinement (physical entrapment), the protein entities can be fully stabilized for storage. Further advances in the field of structural and functional stabilization of protein entities will enable not only their stabilization but also their incorporation into bioreactive coatings, bacterial biosensing devices, biosensors, and biocatalysts.

Having in mind that all protein entities share common features related to their intrinsic instability when put in environments far from physiological conditions, and because the latest advances in biopharmaceutical production point towards an increase of use of protein-based pharmaceuticals, full structural and functional stabilization of said biomolecules will be more and more important if they are to meet commercial success.

\section{Acknowledgments}

Financial support to Victor M. Balcão, via an Invited Research Scientist fellowship (FAPESP Ref. No. 2011/51077-8), and project funding (FAPESP Ref. No. 2013/03181-6, Project PneumoPhageKill) by Fundação de Amparo à Pesquisa do Estado de São Paulo (FAPESP, São Paulo, Brazil), is hereby gratefully acknowledged. The authors are also grateful to Cláudio M. Barroso (BSc.), Graphic Designer at University of Sorocaba (UNISO), for computer-designing the schemes/drawings integrating this review paper.

\section{References}

[1] W. Klonowski, Non-equilibrium proteins, Comput. Chem. 25 (2001) 349-368.

[2] É. Tüdos, A. Fiser, Á. Simon, Z. Dosztányi, M. Fuxreiter, C. Magyar, I. Simon, Noncovalent cross-links in context with other structural and functional elements of proteins, J. Chem. Inf. Comput. Sci. 44 (2004) 347-351.

[3] P.V. Iyer, L. Ananthanarayan, Enzyme stability and stabilization - aqueous and non-aqueous environment, Process Biochem. 43 (2008) 1019-1032.

[4] V. Villegas, A.R. Viguera, F.X. Avilés, L. Serrano, Stabilization of proteins by rational design of alpha-helix stability using helix/coil transition theory, Fold. Des. 01 (1995) 29-34.

[5] R. Wimmer, M. Olsson, M.T.N. Petersen, R. Hatti-Kaul, S.B. Petersen, N. Müller, Towards a molecular level understanding of protein stabilization: the interaction between lysozyme and sorbitol, J. Biotechnol. 55 (1997) 85-100.

[6] W. Wang, Instability, stabilization, and formulation of liquid protein pharmaceuticals, Int. J. Pharm. 185 (1999) 129-188.

[7] J.M. Sanchez-Ruiz, Protein kinetic stability, Biophys. Chem. 148 (2010) 1-15.
[8] R. Fernández-Lafuente, Stabilization of multimeric enzymes: strategies to prevent subunit dissociation, Enzym. Microb. Technol. 45 (2009) 405-418.

[9] N.R. Maddux, S.B. Joshi, D.B. Volkin, J.P. Ralston, C.R. Middaugh, Multidimensiona methods for the formulation of biopharmaceuticals and vaccines, J. Pharm. Sci. 100 (2011) 4171-4197.

[10] V.M. Balcão, T.A. Oliveira, F.X. Malcata, Stability of a commercial lipase from Mucor javanicus: kinetic modelling of $\mathrm{pH}$ and temperature dependencies, Biocatal Biotransf. 16 (1998) 45-66.

[11] I. Serra, C.D. Serra, S. Rocchietti, D. Ubiali, M. Terreni, Stabilization of thymidine phosphorylase from Escherichia coli by immobilization and post immobilization techniques, Enzym. Microb. Technol. 49 (2011) 52-58.

[12] T. Arakawa, S.J. Prestrelski, W.C. Kenney, J.F. Carpenter, Factors affecting short-term and long-term stabilities of proteins, Adv. Drug Deliv. Rev. 10 (1993) 1-28.

[13] T. Arakawa, S.J. Prestrelski, W.C. Kenney, J.F. Carpenter, Factors affecting short-term and long-term stabilities of proteins, Adv. Drug Deliv. Rev. 46 (2001) 307-326.

[14] C.G. Moutinho, C.M. Matos, J.A. Teixeira, V.M. Balcão, Nanocarrier possibilities for functional targeting of bioactive peptides and proteins: state-of-the-art, J. Drug Target. 20 (2012, Feb.) 114-141.

[15] V.M. Balcão, C.G. Moutinho, Peptides and proteins: non-invasive delivery, in: J. Swarbrick (Ed.), 4th edition, Encyclopedia of Pharmaceutical Science and Technology, Vol. IV, CRC Press (Taylor \& Francis Group LLC), 2013, pp. 2555-2577.

[16] V.V. Mozhaev, K. Martinek, Structure-stability relationships in proteins: a guide to approaches to stabilizing enzymes, Adv. Drug Deliv. Rev. 4 (1990) 387-419.

[17] I.G. Denisov, Thermal stability of proteins in intermolecular complexes, Biophys. Chem. 44 (1992) 71-75.

[18] L. Foit, G.J. Morgan, M.J. Kern, L.R. Steimer, A.A. von Hacht, J. Titchmarsh, S.L. Warriner, S.E. Radford, J.C.A. Bardwell, Optimizing protein stability in vivo, Mol. Cell 36 (2009) 861-871.

[19] V. Ragoonanan, A. Aksan, Protein stabilization, Transfus. Med. Hemother. 34 (2007) 246-252.

[20] S.B. Petersen, P.H. Jonson, P. Fojan, E.I. Petersen, M.T.N. Petersen, S. Hansen, R.J Ishak, E. Hough, Protein engineering the surface of enzymes, J. Biotechnol. 66 (1998) 11-26.

[21] C. Magyar, É. Tüdös, I. Simon, Functionally and structurally relevant residues of enzymes: are they segregated or overlapping? FEBS Lett. 567 (2004) 239-242.

[22] C. Scharnagl, M. Reif, J. Friedrich, Stability of proteins: temperature, pressure and the role of the solvent, Biochim. Biophys. Acta 1749 (2005) 187-213.

[23] S.R. Shenoy, B. Jayaram, Proteins: sequence to structure and function - current status, Curr. Protein Pept. Sci. 11 (2010) 498-514.

[24] A. Illanes, A. Cauerhff, L. Wilson, G.R. Castro, Recent trends in biocatalysis engineering, Bioresour. Technol. 115 (2012) 48-57.

[25] S.B. Jadhav, R.S. Singhal, Conjugation of alpha-amylase with dextran for enhanced stability: process details, kinetics and structural analysis, Carbohydr. Polym. 90 (2012) 1811-1817.

[26] C. Berezin, F. Glaser, J. Rosenberg, I. Paz, T. Pupko, P. Fariselli, R. Casadio, N. Ben-Tal, ConSeq: the identification of functionally and structurally important residues in protein sequences, Bioinformatics 20 (2004) 1322-1324.

[27] R. Jaenicke, Protein stability and molecular adaptation to extreme conditions, Eur. J Biochem. 202 (1991) 715-728.

[28] C. Vieille, J.G. Zeikus, Thermoenzymes: identifying molecular determinants of protein structural and functional stability, Trends Biotechnol. 14 (1996) 183-191.

[29] J. Damborsky, Quantitative structure-function and structure-stability relationships of purposely modified proteins, Protein Eng. 11 (1998) 21-30.

[30] W.J. Becktel, J.A. Schellman, Protein stability curves, Biopolymers 26 (1987) 1859-1877.

[31] G.N. Somero, Proteins and temperature, Annu. Rev. Physiol. 57 (1995) 43-68.

[32] B. Lee, G. Vasmatzis, Stabilization of protein structures, Curr. Opin. Biotechnol. 8 (1997) 423-428.

[33] V.P. Torchilin, A.V. Maksimenko, V.N. Smirnov, I.V. Berezin, A.M. Klibanov, K. Martinek, The principles of enzyme stabilization. IV. Modification of "key" functional groups in the tertiary structure of proteins, Biochim. Biophys. Acta 567 (1979) 1-11.

[34] M.M. Kristjánsson, J.E. Kinsella, Protein and enzyme stability: structural, thermodynamic, and experimental aspects, Adv. Food Nutr. Res. 35 (1991) 237-316.

[35] H. Park, S. Lee, Prediction of the mutation-induced change in thermodynamic stabilities of membrane proteins from free energy simulations, Biophys. Chem. 114 (2005) 191-197.

[36] B.K. Shoichet, W.A. Baase, R. Kuroki, B.W. Matthews, A relationship between protein stability and protein function, Proc. Natl. Acad. Sci. U. S. A. 92 (1995) 452-456.

[37] C. Ó'Fágáin, Enzyme stabilization - recent experimental progress, Enzym. Microb. Technol. 33 (2003) 137-149.

[38] R.M. Bhaskara, N. Srinivasan, Stability of domain structures in multi-domain proteins, Sci. Reports 1 (2011) 40-48.

[39] L.A. Campos, M.M. Garcia-Mira, R. Godoy-Ruiz, J.M. Sanchez-Ruiz, J. Sancho, Do proteins always benefit from a stability increase? Relevant and residual stabilization in a three-state protein by charge optimization, J. Mol. Biol. 344 (2004) 223-237.

[40] G. Xie, S.N. Timasheff, The thermodynamic mechanism of protein stabilization by trehalose, Biophys. Chem. 64 (1997) 25-43.

[41] T.C. Jyothi, S. Sinha, S.A. Singh, A. Surolia, A.G.A. Rao, Napin from Brassica juncea: thermodynamic and structural analysis of stability, Biochim. Biophys. Acta 1774 (2007) 907-919.

[42] T.R. Foss, M.S. Kelker, R.L. Wiseman, I.A. Wilson, J.W. Kelly, Kinetic stabilization of the native state by protein engineering: implications for inhibition of transthyretin amyloidogenesis, J. Mol. Biol. 347 (2005) 841-854.

[43] B. Palmer, K. Angus, L. Taylor, J. Warwicker, J.P. Derrick, Design of stability at extreme alkaline pH in streptococcal protein G, J. Biotechnol. 134 (2008) 222-230. 
[44] G. Castronuovo, Proteins in aqueous solutions. Calorimetric studies and thermodynamic characterization, Thermochim. Acta 193 (1991) 363-390.

[45] C. Mateo, B.C.C. Pessela, M. Fuentes, R. Torres, L. Betancor, A. Hidalgo, G. FernándezLorente, R. Fernández-Lafuente, J.M. Guisán, Stabilization of multimeric enzymes via immobilization and further cross-linking with aldehyde-dextran, chapter 12 , in: Second edition, J.M. Guisán (Ed.), Methods in Biotechnology - Immobilization of Enzymes and Cells, Vol. 22, Humana Press Inc., Totowa NJ, USA, 2006, pp. $129-141$.

[46] F. López-Gallego, L. Betancor, A. Hidalgo, G. Dellamora-Ortiz, C. Mateo, R. Fernández-Lafuente, J.M. Guisán, Stabilization of different alcohol oxidases via immobilization and post immobilization techniques, Enzym. Microb. Technol. 40 (2007) 278-284.

[47] G.I. Makhatadze, P.L. Privalov, Contribution of hydration to protein folding thermodynamics: I. The enthalpy of hydration, J. Mol. Biol. 232 (1993) 639-659.

[48] Y.-H. Liao, M.B. Brown, G.P. Martin, Investigation of the stabilization of freeze-dried lysozyme and the physical properties of the formulations, Eur. J. Pharm. Biopharm. 58 (2004) 15-24

[49] O. Miyawaki, Hydration state change of proteins upon unfolding in sugar solutions, Biochim. Biophys. Acta 1774 (2007) 928-935.

[50] K.P. Murphy, V. Bhakuni, D. Xie, E. Freire, Molecular basis of co-operativity in protein folding. III. Structural identification of cooperative folding units and folding intermediates, J. Mol. Biol. 227 (1992) 293-306.

[51] J.K. Cheung, P. Shah, T.M. Truskett, Heteropolymer collapse theory for protein folding in the pressure-temperature plane, Biophys. J. 91 (2006) 2427-2435.

[52] S.A. Potekhin, A.A. Senin, N.N. Abdurakhmanov, E.I. Tiktopulo, High pressure stabilization of collagen structure, Biochim. Biophys. Acta 1794 (2009) 1151-1158.

[53] J.K. Myers, S.R. Trevino, Increasing globular protein stability, Chem. Today 30 (2012) 30-33.

[54] A.R. Bizzarri, S. Cannistraro, Molecular dynamics of water at the protein-solvent interface, J. Phys. Chem. B 106 (2002) 6617-6633.

[55] O. Miyawaki, Thermodynamic analysis of protein unfolding in aqueous solutions as a multisite reaction of protein with water and solute molecules, Biophys. Chem. 144 (2009) 46-52.

[56] P.W. Fenimore, H. Frauenfelder, B.H. McMahon, F.G. Parak, Slaving: solvent fluctuations dominate protein dynamics and functions, Proc. Natl. Acad. Sci. U. S. A. 99 (2002) 16047-16051.

[57] W. Doster, M. Settles, Protein-water displacement distributions, Biochim. Biophys. Acta 1749 (2005) 173-186.

[58] A. Luzar, Water-hydrogen bond dynamics close to hydrophobic and hydrophilic groups, Faraday Discuss. 103 (1996) 29-40.

[59] E.L. Kovrigin, S.A. Potekhin, On the stabilizing action of protein denaturants: acetonitrile effect on stability of lysozyme in aqueous solutions, Biophys. Chem. 83 (2000) 45-59.

[60] W. Wang, Protein aggregation and its inhibition in biopharmaceutics, Int. J. Pharm 289 (2005) 1-30.

[61] M.R. Ganjalikhany, B. Ranjbar, S. Hosseinkhani, K. Khalifeh, L. Hassani, Roles of trehalose and magnesium sulfate on structural and functional stability of firefly luciferase, J. Mol. Catal. B Enzym. 62 (2010) 127-132.

[62] J. Kohda, H. Kawanishi, K.-I. Suehara, Y. Nakano, T. Yano, Stabilization of free and immobilized enzymes using hyperthermophilic chaperonin, J. Biosci. Bioeng. 101 (2006) 131-136.

[63] I. Sakane, M. Ikeda, C. Matsumoto, T. Higurashi, K. Inoue, K. Hongo, T. Mizobata, Y. Kawata, Structural stability of oligomeric chaperonin 10: the role of two betastrands at the $\mathrm{N}$ and C termini in structural stabilization, J. Mol. Biol. 344 (2004) 1123-1133.

[64] Y.-H. Wong, S. Tayyab, Protein stabilizing potential os simulated honey sugar cocktail under various denaturation conditions, Process Biochem. 47 (2012) 1933-1943.

[65] J.N. Talbert, J.M. Goddard, Enzymes on material surfaces, Colloids Surf. B: Biointerfaces 93 (2012) 8-19.

[66] L. Pauling, R.B. Corey, H.R. Branson, The structure of proteins: two hydrogenbonded helical configurations of the polypeptide chain, Proc. Natl. Acad. Sci. U. S. A. 37 (1951) 205-211.

[67] D. Shah, T.P. Johnston, A.K. Mitra, Thermodynamic parameters associated with guanidine $\mathrm{HCl}$ - and temperature-induced unfolding of bFGF, Int. J. Pharm. 169 (1998) 1-14

[68] C. Cléry-Barraud, F. Renault, J. Leva, N. El Bakdouri, P. Masson, D. Rochu, Exploring the structural and functional stabilities of different paraoxonase- 1 formulations through electrophoretic mobilities and enzyme activity parameters under hydrostatic pressure, Biochim. Biophys. Acta 1794 (2009) 680-688.

[69] O. Miyawaki, M. Tatsuno, Thermodynamic analysis of alcohol effect on thermal stability of proteins, J. Biosci. Bioeng. 111 (2011) 198-203.

[70] R. Jaenicke, G. Bohm, The stability of proteins in extreme environments, Curr. Opin. Struct. Biol. 8 (1998) 738-748.

[71] F. Kong, Y.F. Hu, Biomolecule immobilization techniques for bioactive paper fabrication, Anal. Bioanal. Chem. 403 (2012) 7-13.

[72] V.M. Balcão, A.F. Azevedo, L.M. Castro, C.I. Costa, S. Santos, C.M. Matos, C.G. Moutinho, J.A. Teixeira, J.C. Azeredo, Design of a lipid nanovesicle system encapsulating bacteriophages integrated in a multiple emulsion formulation: a proof-ofconcept, in: Nanotech 2010 NSTI (Ed.), Technical Proceedings of the 2010 NST Nanotechnology Conference \& Expo (June 21-24, 2010, Anaheim CA, USA) Chapter 7, Anaheim CA, Vol. 3, 2010, pp. 459-462.

[73] V.M. Balcão, A.R. Moreira, C.G. Moutinho, M.V. Chaud, M. Tubino, M.M. Vila, Structural and functional stabilization of phage particles in carbohydrate matrices for bacterial biosensing, Enzym. Microb. Technol. 53 (2013) 55-69.

[74] A. Graf, K.S. Jack, A.K. Whittaker, S.M. Hook, T. Rades, Protein delivery using nanoparticles based on microemulsions with different structure-types, Eur. J. Pharm. Sci. 33 (2008) 434-444.
[75] V.P. Torchilin, Cell penetrating peptide-modified pharmaceutical nanocarriers for intracellular drug and gene delivery, Biopolymers 90 (2008) 604-610.

[76] V.P. Torchilin, Tat peptide-mediated intracellular delivery of pharmaceutical nanocarriers, Adv. Drug Deliv. Rev. 60 (2008) 548-558.

[77] A. Cooper, Thermodynamic consequences of the removal of a disulfide bridge from hen lysozyme, J. Mol. Biol. 225 (1992) 939-943.

[78] B.I. Dahiyat, In silico design for protein stabilization, Curr. Opin. Biotechnol. 10 (1999) 387-390.

[79] N.N. Khechinashvili, Thermodynamic properties of globular proteins and the principle of stabilization of their native structure, Biochim. Biophys. Acta 1040 (1990) 346-354.

[80] P.L. Privalov, Thermodynamic bases of the stability of protein structure, Thermochim. Acta 163 (1990) 33-46.

[81] N.N. Khechinashvili, S.A. Volchkov, A.V. Kabanov, G. Barone, Thermal stability of proteins does not correlate with the energy of intramolecular interactions, Biochim. Biophys. Acta 1784 (2008) 1830-1834.

[82] H.-J. Hinz, C. Steif, T. Vogl, R. Meyer, M. Renner, R. Ledermüller, Fundamentals of protein stability, Pure Appl. Chem. 65 (1993) 947-952.

[83] T. Arakawa, D. Ejima, Y. Kita, K. Tsumoto, Small molecule pharmacological chaperones: from thermodynamic stabilization to pharmaceutical drugs, Biochim. Biophys. Acta 1764 (2006) 1677-1687.

[84] D. Zhou, G.G.Z. Zhang, D. Law, D.J.W. Grant, E.A. Schmitt, Physical stability of amorphous pharmaceuticals: importance of configurational thermodynamic quantities and molecular mobility, J. Pharm. Sci. 91 (2002) 1863-1872.

[85] S.P. Duddu, G. Zhang, P.R. Dal Monte, The relationship between protein aggregation and molecular mobility below the glass transition temperature of lyophilized formulations containing a monoclonal antibody, Pharm. Res. 14 (1997) 596-600.

[86] G.I. Makhatadze, P.L. Privalov, Protein interactions with urea and guadinium chloride. A calorimetric study, J. Mol. Biol. 226 (1992) 491-505.

[87] L. Lavelle, J.R. Fresco, Stabilization of nucleic acid triplexes by high concentrations of sodium and ammonium salts follows the Hofmeister series, Biophys. Chem. 105 (2003) 681-699.

[88] X. Tang, M.J. Pikal, Measurement of the kinetics of protein unfolding in viscous systems and implications for protein stability in freeze-drying, Pharm. Res. 22 (2005) 1176-1185.

[89] T. Kamiyama, Y. Sadahide, Y. Nogusa, K. Gekko, Polyol-induced molten globule of cytochrome $c$ : an evidence for stabilization by hydrophobic interaction, Biochim. Biophys. Acta 1434 (1999) 44-57.

[90] I. Haque, R. Singh, A.A. Moosavi-Movahedi, F. Ahmad, Effect of polyol osmolytes on DGD, the Gibbs energy of stabilization of proteins at different pH values, Biophys. Chem. 117 (2005) 1-12

[91] I. Haque, R. Singh, F. Ahmad, A.A. Moosavi-Movahedi, Testing polyols' compatibility with Gibbs energy of stabilization of proteins under conditions in which they behave as compatible osmolytes, FEBS Lett. 579 (2005) 3891-3898.

[92] D. Rodriguez-Larrea, S. Minning, T.V. Borchert, J.M. Sanchez-Ruiz, Role of solvation barriers in protein kinetic stability, J. Mol. Biol. 360 (2006) 715-724.

[93] B.A. Kelch, D.A. Agard, Mesophile versus thermophile: insights into the structural mechanisms of kinetic stability, J. Mol. Biol. 370 (2007) 784-795.

[94] T.J. Ahern, A.M. Klibanov, The mechanism of irreversible enzyme inactivation at $100{ }^{\circ} \mathrm{C}$, Science 228 (1985) 1280-1284

[95] V.M. Balcão, R. Fernandez-Lafuente, F.X. Malcata, J.M. Guisán, Structural and functional stabilization of L-asparaginase from Escherichia coli upon immobilization onto highly activated supports: possible biomedical applications using extracorporeal bioreactors, Electron. J. Biotechnol. (2000) (http://www.ejbiotechnology.info/ feedback/proceedings/04/poster/p66.html).

[96] V.M. Balcão, C. Mateo, R. Fernández-Lafuente, F.X. Malcata, J.M. Guisán, Structural and functional stabilization of L-asparaginase via multi-subunit immobilization onto highly activated supports, Biotechnol. Prog. 17 (2001) 537-542.

[97] V.M. Balcão, C. Mateo, R. Fernández-Lafuente, F.X. Malcata, J.M. Guisán, Coimmobilization of L-asparaginase and glutamate dehydrogenase onto highly activated supports, Enzym. Microb. Technol. 28 (2001) 696-704.

[98] R. Jaenicke, Glyceraldehyde-3-phosphate dehydrogenase from Thermotoga maritima: strategies of protein stabilization, FEMS Microbiol. Rev. 18 (1996) 215-224.

[99] R. Jaenicke, H. Schurig, N. Beaucamp, R. Ostendorp, Structure and stability of hyperstable proteins: glycolytic enzymes from hyperthermophilic bacterium Thermotoga maritima, Adv. Protein Chem. 48 (1996) 181-269.

[100] R. Das, M. Gerstein, The stability of thermophilic proteins: a study based on comprehensive genome comparison, Funct. Integr. Genomics 1 (2000) 76-88.

[101] R. Jaenicke, Stability and stabilization of globular proteins in solution, J. Biotechnol. 79 (2000) 193-203.

[102] D.F.M. Neri, V.M. Balcão, M.G. Carneiro-da-Cunha, L.B. Carvalho Jr., J.A. Teixeira, Immobilization of $\beta$-galactosidase from Kluyveromyces lactis onto a polysiloxanepolyvinyl alcohol magnetic (mPOS-PVA) composite for lactose hydrolysis, Catal. Commun. 9 (2008) 2334-2339.

[103] D.F.M. Neri, V.M. Balcão, R.S. Costa, E.C. Ferreira, D.P.M. Torres, L.R. Rodrigues, L.B. Carvalho Jr., J.A. Teixeira, $\beta$-Galactosidase from Aspergillus oryzae immobilized onto different magnetic supports: a comparative experimental and modelling study of the galactooligosaccharides production, in: E.C. Ferreira, M. Mota (Eds.), Proceedings of the 10th International Chemical and Biological Engineering Conference - CHEMPOR 2008, Braga, Portugal, September 4-6, 2008, pp. 1036-1041.

[104] D.F.M. Neri, V.M. Balcão, F.O.Q. Dourado, J.M.B. Oliveira, L.B. Carvalho Jr., J.A. Teixeira, Galactooligosaccharides production by $\beta$-galactosidase immobilized onto magnetic polysiloxane-polyaniline particles, React. Funct. Polym. 69 (2009) 246-251. 
[105] D.F.M. Neri, V.M. Balcão, D.P.M. Torres, L.M. Rodrigues, R. Costa, I.C.A.P. Rocha, E.M.F.C. Ferreira, L.B. Carvalho Jr., J.A. Teixeira, Galacto-oligosaccharides production during lactose hydrolysis by free Aspergillus oryzae $\beta$-galactosidase and immobilized on magnetic polysiloxane-polyvinyl alcohol, Food Chem. 115 (2009) 92-99.

[106] D.F.M. Neri, V.M. Balcão, F.O.Q. Dourado, J.M.B. Oliveira, L.B. Carvalho Jr., J.A. Teixeira, Immobilized beta-galactosidase onto magnetic particles coated with polyaniline: support characterization and galactooligosaccharides production, J. Mol. Catal. B Enzym. 70 (2011) 74-80.

[107] D.F.M. Neri, V.M. Balcão, S.M. Cardoso, A.M.S. Silva, M.R.M. Domingues, D.P.M. Torres, L.R.M. Rodrigues, L.B. Carvalho Jr., J.A. Teixeira, Characterization of galactooligosaccharides produced by beta-galactosidase immobilized onto magnetized Dacron, Int. Dairy J. 21 (2011) 172-178.

[108] C. Rocha, V.M. Balcão, M.P. Gonçalves, J.A. Teixeira, Spent grain as a new carrier for trypsin immobilisation, Proceedings of the 34th International Conference of Slovak Society of Chemical Engineering (SSCHE), 2007, pp. 277-284.

[109] S. Arola, T. Tammelin, H. Setälä, A. Tullila, M.B. Linder, Immobilization-stabilization of proteins on nanofibrillated cellulose derivatives and their bioactive film formation, Biomacromolecules 13 (2012) 594-603.

[110] J.M. Bolivar, J. Rocha-Martín, C. Mateo, J.M. Guisán, Stabilization of a highly active but unstable alcohol dehydrogenase from yeast using immobilization and postimmobilization techniques, Process Biochem. 47 (2012) 679-686.

[111] V. Grazu, F. López-Gallego, J.M. Guisán, Tailor-made design of penicillin G acylase surface enables its site-directed immobilization and stabilization onto commercial mono-functional epoxy supports, Process Biochem. 47 (2012) 2538-2541.

[112] K.E.S. Tang, K.A. Dill, Native protein fluctuations: the conformational-motion temperature and the inverse correlation of protein flexibility with protein stability, J. Biomol. Struct. Dyn. 16 (1998) 397-411.

[113] B.C. Pessela, C. Mateo, M. Filho, A.V. Carrascosa, R. Fernández-Lafuente, J.M. Guisán Stabilization of the quaternary structure of a hexameric alpha-galactosidase from Thermus sp. T2 by immobilization and post-immobilization techniques, Process Biochem. 43 (2008) 193-198.

[114] C. Bernal, L. Sierra, M. Mesa, Improvement of thermal stability of beta-galactosidase from Bacillus circulans by multipoint covalent immobilization in hierarchical macro-mesoporous silica, J. Mol. Catal. B Enzym. 84 (2012) 166-172.

[115] B. Boscolo, F. Trotta, E. Ghibaudi, High catalytic performances of Pseudomonas fluorescens lipase adsorbed on a new type of cyclodextrin-based nanosponges, J. Mol. Catal. B Enzym. 62 (2010) 155-161.

[116] V.M. Balcão, A.L. Paiva, F.X. Malcata, Bioreactors with immobilized lipases: state-ofthe-art, Enzym. Microb. Technol. 18 (1996) 392-416.

[117] D.A. Cowan, R. Fernández-Lafuente, Enhancing the functional properties of thermophilic enzymes by chemical modification and immobilization, Enzym. Microb. Technol. 49 (2011) 326-346.

[118] V.M. Balcão, C.I. Costa, C.M. Matos, C.G. Moutinho, M. Amorim, M.E. Pintado, A.P. Gomes, M.M. Vila, J.A. Teixeira, Nanoencapsulation of bovine lactoferrin for food and biopharmaceutical applications, Food Hydrocoll. 32 (2013) 425-431.

[119] F. López-Gallego, L. Betancor, A. Hidalgo, C. Mateo, J.M. Guisán, R. FernándezLafuente, Optimization of an industrial biocatalyst of glutaryl acylase: stabilization of the enzyme by multipoint covalent attachment onto new amino-epoxy Sepabeads, J. Biotechnol. 111 (2004) 219-227.

[120] G. Czichocki, H. Dautzenberg, E. Capan, K.-D. Vorlop, New and effective entrapment of polyelectrolyte-enzyme-complexes in LentiKats, Biotechnol. Lett. 23 (2001) 1303-1307.

[121] M. Schlieker, K.-D. Vorlop, A novel immobilization method for entrapment: LentiKats ${ }^{\circledR}$, chapter 29, Methods in Biotechnology - Immobilization of Enzymes and Cells, Vol. 22, 2006, pp. 333-343.

[122] J.A. Figueira, H.H. Sato, P. Fernandes, Establishing the feasibility of using $\beta$ glucosidase entrapped in Lentikats and in sol-gel supports for cellobiose hydrolysis, J. Agric. Food Chem. 61 (2013) 626-634.

[123] M. Fuentes, C. Mateo, J.M. Guisán, R. Fernández-Lafuente, Preparation of inert magnetic nano-particles for the directed immobilization of antibodies, Biosens. Bioelectron. 20 (2005) 1380-1387.

[124] M.C. Flickinger, J.L. Schottel, D.R. Bond, A. Aksan, L.E. Scriven, Painting and printing living bacteria: engineering nanoporous biocatalytic coatings to preserve microbial viability and intensify reactivity, Biotechnol. Prog. 23 (2007) 2-17.

[125] M. Yoshimoto, H. Sakamoto, H. Shirakami, Covalent conjugation of tetrameric bovine liver catalase to liposome membranes for stabilization of the enzyme tertiary and quaternary structures, Colloids Surf. B: Biointerfaces 69 (2009) 281-287.

[126] V.M. Balcão, M.C. Vieira, F.X. Malcata, Adsorption of protein from several commercial lipase preparations onto a hollow-fiber membrane module, Biotechnol. Prog. 12 (1996) 164-172.

[127] V.M. Balcão, F.X. Malcata, On the performance of a hollow-fiber bioreactor for acidolysis catalyzed by immobilized lipase, Biotechnol. Bioeng. 60 (1998) 114-123.

[128] A.L. Paiva, V.M. Balcão, F.X. Malcata, Kinetics and mechanisms of reactions catalyzed by immobilized lipases, Enzym. Microb. Technol. 27 (2000) 187-204.

[129] C. Mateo, J.M. Palomo, G. Fernández-Lorente, J.M. Guisán, R. Fernández-Lafuente, Improvement of enzyme activity, stability and selectivity via immobilization techniques, Enzym. Microb. Technol. 40 (2007) 1451-1463.

[130] R. Verger, "Interfacial activation" of lipases: facts and artifacts, Trends Biotechnol. 15 (1997) 32-38.

[131] P. Grunwald, R. Freder, W. Gunßer, Application of polyethylene imine as carrier material for enzyme immobilization, Naturwissenschaften 68 (1981) 525-526.

[132] M. Fuentes, B.C. Pessela, J.V. Maquiese, C. Ortiz, R.L. Segura, J.M. Palomo, O. Abian, R. Torres, C. Mateo, R. Fernández-Lafuente, J.M. Guisán, Reversible and strong immobilization of proteins by ionic exchange on supports coated with sulfate-dextran, Biotechnol. Prog. 20 (2004) 1134-1139.
[133] J.M. Bolivar, C. Mateo, J. Rocha-Martin, F. Cava, J. Berenguer, R. Fernández-Lafuente, J.M. Guisán, The adsorption of multimeric enzymes on very lowly activated supports involves more enzyme subunits: stabilization of a glutamate dehydrogenase from Thermus thermophilus by immobilization on heterofunctional supports, Enzym. Microb. Technol. 44 (2009) 139-144.

[134] B.K. Vaidya, R.S. Singhal, Use of insoluble yeast beta-glucan as a support for immobilization of Candida rugosa lipase, Colloids Surf. B: Biointerfaces 61 (2008) 101-105.

[135] T. Serno, R. Geidobler, G. Winter, Protein stabilization by cyclodextrins in the liquid and dried state, Adv. Drug Deliv. Rev. 63 (2011) 1086-1106.

[136] R.M. Barros, C.I. Extremina, I.C. Gonçalves, B.O. Braga, V.M. Balcão, F.X. Malcata, Hydrolysis of $\alpha$-lactalbumin by cardosin A immobilized on highly activated supports, Enzym. Microb. Technol. 33 (2003) 908-916.

[137] F. López-Gallego, L. Betancor, C. Mateo, A. Hidalgo, N. Alonso-Morales, G. Dellamora-Ortiz, J.M. Guisán, R. Fernández-Lafuente, Enzyme stabilization by glutaraldehyde crosslinking of adsorbed proteins on aminated supports, J. Biotechnol. 119 (2005) 70-75.

[138] C. Mateo, J.M. Palomo, M. Fuentes, L. Betancor, V. Grazu, F. López-Gallego, B.C.C. Pessela, A. Hidalgo, G. Fernández-Lorente, R. Fernández-Lafuente, J.M. Guisán, Glyoxyl agarose: a fully inert and hydrophilic support for immobilization and high stabilization of proteins, Enzym. Microb. Technol. 39 (2006) 274-280.

[139] C. Mateo, O. Abian, M. Bernedo, E. Cuenca, M. Fuentes, G. Fernandez-Lorente, J.M. Palomo, V. Grazu, B.C.C. Pessela, C. Giacomini, G. Irazoqui, A. Villarino, K. Ovsejevi, F. Batista-Viera, R. Fernandez-Lafuente, J.M. Guisán, Some special features of glyoxyl supports to immobilize proteins, Enzym. Microb. Technol. 37 (2005) 456-462.

[140] R. Fernández-Lafuente, V. Rodŕíguez, C. Mateo, G. Penzol, O. Hernández-Justiz, G. Irazoqui, A. Villarino, K. Ovsejevi, F. Batista, J.M. Guisán, Stabilization of multimeric enzymes via immobilization and post-immobilization techniques, J. Mol. Catal. B Enzym. 7 (1999) 181-189.

[141] O. Abian, L. Wilson, C. Mateo, G. Fernández-Lorente, J.M. Palomo, R. FernándezLafuente, J.M. Guisán, D. Re, A. Tam, M. Daminatti, Preparation of artificial hyperhydrophilic micro-environments (polymeric salts) surrounding enzyme molecules: new enzyme derivatives to be used in any reaction medium, J. Mol. Catal. B Enzym. 19-20 (2002) 295-303.

[142] R. Fernández-Lafuente, C. Rosell, V. Rodriguez, J.M. Guisán, Strategies for enzyme stabilization by intramolecular crosslinking with bifunctional reagents, Enzym. Microb. Technol. 17 (1995) 517-523.

[143] J.M. Bolivar, J. Rocha-Martin, C. Mateo, F. Cava, J. Berenguer, R. Fernandéz-Lafuente, J.M. Guisán, Coating of soluble and immobilized enzymes with ionic polymers: full stabilization of the quaternary structure of multimeric enzymes, Biomacromolecules 10 (2009) 742-747.

[144] J.F. Celayeta, A.H. Silva, V.M. Balcão, F.X. Malcata, Maximisation of the yield of final product on substrate in the case of sequential reactions catalysed by coimmobilised enzymes: a theoretical analysis, Bioprocess Biosyst. Eng. 24 (2001) 143-149.

[145] E.M. Lamas, R.M. Barros, V.M. Balcão, F.X. Malcata, Hydrolysis of whey proteins by proteases extracted from Cynara cardunculus and immobilized onto highly activated supports, Enzym. Microb. Technol. 28 (2001) 642-652.

[146] G.A. Kotzia, N.E. Labrou, L-asparaginase from Erwinia chrysanthemi 3937: cloning expression and characterization, J. Biotechnol. 127 (2007) 657-669.

[147] J.M. Bolivar, F. López-Gallego, C. Godoy, D.S. Rodrigues, R.C. Rodrigues, P. Batalla, J Rocha-Martín, C. Mateo, R.L.C. Giordano, J.M. Guisán, The presence of thiolated compounds allows the immobilization of enzymes on glyoxyl agarose at mild $\mathrm{pH}$ values: new strategies of stabilization by multipoint covalent attachment, Enzym. Microb. Technol. 45 (2009) 477-483.

[148] C.-Y. Tan, C.-H. Xu, K.-C. Ruan, Folding studies of two hydrostatic pressure sensitive proteins, Biochim. Biophys. Acta 1764 (2006) 481-488.

[149] H.J. Lee, A. McAuley, K.F. Schilke, J. McGuire, Molecular origins of surfactantmediated stabilization of protein drugs, Adv. Drug Deliv. Rev. 63 (2011) 1160-1171.

[150] R.K. Eppler, R.S. Komor, J. Huynh, J.S. Dordick, J.A. Reimer, D.S. Clark, Water dynamics and salt-activation of enzymes in organic media: mechanistic implications revealed by NMR spectroscopy, Proc. Natl. Acad. Sci. U. S. A. 103 (2006) 5706-5710.

[151] R. Lumry, H. Eyring, Conformation changes of proteins, J. Phys. Chem. 58 (1954) $110-120$.

[152] C. Ebel, H. Eisenberg, R. Ghirlando, Probing protein-sugar interactions, Biophys. J. 78 (2000) 385-393.

[153] K. Sasahara, P. McPhie, A.P. Minton, Effect of dextran on protein stability and conformation attributed to macromolecular crowding, J. Mol. Biol. 326 (2003) 1227-1237.

[154] V. Kumar, R. Chari, V.K. Sharma, D.S. Kalonia, Modulation of the thermodynamic stability of proteins by polyols: significance of polyol hydrophobicity and impact on the chemical potential of water, Int. J. Pharm. 413 (2011) 19-28.

[155] J.F. Carpenter, J.H. Crowe, The mechanism of cryoprotection of proteins by solutes, Cryobiology 25 (1988) 244-255.

[156] F. Anjum, V. Rishi, F. Ahmad, Compatibility of osmolytes with Gibbs energy of stabilization of proteins, Biochim. Biophys. Acta 1476 (2000) 75-84.

[157] T.F. O'Connor, P.G. Debenedetti, J.D. Carbeck, Stability of proteins in the presence of carbohydrates; experiments and modeling using scaled particle theory, Biophys. Chem. 127 (2007) 51-63.

[158] I. Julca, M. Alaminos, J. González-López, M. Manzanera, Xeroprotectants for the stabilization of biomaterials, Biotechnol. Adv. 30 (2012) 1641-1654.

[159] J.H. Crowe, L.M. Crowe, R. Mouradian, Stabilization of biological membranes at low water activities, Cryobiology 20 (1983) 346-356.

[160] J.S. Clegg, The physical properties and metabolic status of Artemia cysts at low water contents: the water replacement hypothesis, in: A. Carl Leopold (Ed.), 
Membranes, Metabolism and Dry Organisms, Cornell University Press, 1986, pp. 169-187.

[161] W. Saenger, Structure and dynamics of water surrounding biomolecules, Annu. Rev. Biophys. Biophys. Chem. 16 (1987) 93-114

[162] A. Cesàro, O. De Giacomo, F. Sussich, Water interplay in trehalose polymorphism, Food Chem. 106 (2008) 1318-1328.

[163] E.A. Golovina, A.V. Golovin, F.A. Hoekstra, R. Faller, Water replacement hypothesis in atomic detail - factors determining the structure of dehydrated bilayer stacks, Biophys. J. 97 (2009) 490-499.

[164] E.A. Galinski, K. Lippert, Novel compatible solutes and their potential application as stabilizers in enzyme technology, in: F. Rodriguez-Valera (Ed.), General and Applied Aspects of Halophilic Bacteria, Plenum Press, New York, 1991, pp. 351-358.

[165] T.J. Anchordoquy, J.F. Carpenter, Polymers protect lactate dehydrogenase during freezedrying by inhibiting dissociation in the frozen state, Arch. Biochem. Biophys 332 (1996) 231-238.

[166] R.S. Kane, P. Deschatelets, G.M. Whitesides, Kosmotropes form the basis of proteinresistant surfaces, Langmuir 19 (2003) 2388-2391.

[167] S.N. Timasheff, T. Arakawa, Mechanism of protein precipitation and stabilization by co-solvents, J. Cryst. Growth 90 (1988) 39-46.

[168] N.K. Poddar, Z.A. Ansari, R.K.B. Singh, A.A. Moosavi-Movahedi, F. Ahmad, Effect of monomeric and oligomeric sugar osmolytes on $\Delta \mathrm{G}_{\mathrm{D}}$, the Gibbs energy of stabilization of the protein at different $\mathrm{pH}$ values: is the sum effect of monosaccharide individually additive in a mixture? Biophys. Chem. 138 (2008) 120-129.

[169] J.K. Kaushik, R. Bhat, Why is trehalose an exceptional protein stabilizer? An analysis of the thermal stability of proteins in the presence of the compatible osmolyte trehalose, J. Biol. Chem. 278 (2003) 26458-26465.

[170] A. Linhananta, S. Hadizadeh, S.S. Plotkin, An effective solvent theory connecting the underlying mechanisms of osmolytes and denaturants for protein stability, Biophys. J. 100 (2011) 459-468.

[171] B.S. Kendrick, B.S. Chang, T. Arakawa, B. Peterson, T.W. Randolph, M.C. Manning, J.F Carpenter, Preferential exclusion of sucrose from recombinant interleukin-1 receptor antagonist: role in restricted conformational mobility and compaction of native state, Proc. Natl. Acad. Sci. U. S. A. 94 (1997) 11917-11922.
[172] D. Hall, A.P. Minton, Macromolecular crowding: qualitative and semiquantitative successes, quantitative challenges, Biochim. Biophys. Acta, Proteins Proteomics 1649 (2003) 127-139.

[173] C. Tanford, Extension of the theory of linked functions to incorporate the effects of protein hydration, J. Mol. Biol. 39 (1969) 539-544.

[174] P.L. Wang, T.P. Johnston, Enhanced stability of two model proteins in an agitated solution environment using poloxamer 407, J. Parenter. Sci. Technol. 47 (1993) 183-189.

[175] S.N. Timasheff, The control of protein stability and association by weak interactions with water: how do solvents affect these processes? Annu. Rev. Biophys. Biomol. Struct. 22 (1993) 67-97.

[176] A. Tiwari, R. Bhat, Stabilization of yeast hexokinase A by polyol osmolytes: correlation with the physicochemical properties of aqueous solutions, Biophys. Chem. 124 (2006) 90-99.

[177] K.A. Dill, Dominant forces in protein folding, Biochemistry 29 (1990) 7133-7155.

[178] H.J.C. Eriksson, W.L.J. Hinrichs, B. van Veen, G.W. Somsen, G.J. de Jong, H.W. Frijlink, Investigations into the stabilization of drugs by sugar classes: I. Tablets prepared from stabilized alkaline phosphatase, Int. J. Pharm. 249 (2002) 59-70.

[179] I.L. Shulgin, E. Ruckenstein, Preferential hydration and solubility of proteins in aqueous solutions of polyethylene glycol, Biophys. Chem. 120 (2006) 188-198.

[180] L. Martín-Banderas, M. Flores-Mosquera, P. Riesco-Chueca, A. Rodríguez-Gil, Á. Cebolla, S. Chávez, A.M. Gañán-Calvo, Flow focusing: a versatile technology to produce size-controlled and specific-morphology microparticles, Small 1 (2005) 2-6.

[181] H. Luo, C. Pozrikidis, Numerical simulation of particle encapsulation due to liquid thread breakup, Comput. Fluids 38 (2009) 564-571.

[182] M.M.D.C. Vila, M. Tubino, G.O. Neto, Determination of salicylate in blood serum by flow-injection with immobilized salicylate hydroxylase, J. AOAC Int. 84 (2001) 1363-1369.

[183] V.M. Balcão, S.V.P. Barreira, T.M. Nunes, M.V. Chaud, M. Tubino, M.M.D.C. Vila, Carbohydrate hydrogels with stabilized phage particles for bacterial biosensing: bacterium diffusion studies, Appl. Biochem. Biotechnol. 172 (2014) 1194-1214. 